\title{
Efficient Adaptive Sampling Methods Based on Deviation Analysis of Piecewise Cubic Spline Interpolation
}

\section{Xi Cheng}

Tsinghua University

long zeng ( $\square$ zenglong@sz.tsinghua.edu.cn )

Tsinghua University

Haoyu Jiang

Tsinghua University

Xueping Liu

Tsinghua University

\section{Research Article}

Keywords: On-machine inspection, NURBS, Piecewise cubic spline interpolation, Adaptive sampling

Posted Date: June 18th, 2021

DOl: https://doi.org/10.21203/rs.3.rs-596989/v1

License: (c) (i) This work is licensed under a Creative Commons Attribution 4.0 International License. Read Full License 


\title{
Efficient adaptive sampling methods based on deviation analysis of piecewise cubic spline interpolation
}

\author{
Xi Cheng a ${ }^{a}$ Long Zeng ${ }^{\text {a* }}$, Haoyu Jiang ${ }^{a}$, Xueping Liu ${ }^{\text {a,b }}$ \\ ${ }^{a}$ Division of Advanced Manufacturing, Tsinghua Shenzhen International Graduate School, Tsinghua \\ University, 518055 Shenzhen, P.R. China \\ ${ }^{\mathrm{b}}$ Department of Mechanical Engineering, Tsinghua University, 100084 Beijing, P.R. China \\ *Corresponding author. \\ E-mails: cheng-x20@mails.tsinghua.edu.cn (Xi Cheng), zenglong@sz.tsinghua.edu.cn (Long Zeng), \\ twisternb@foxmail.com (Haoyu Jiang), liuxp@sz.tsinghua.edu.cn (Xueping Liu).
}

\begin{abstract}
The on-machine inspection technique requires a certain manufacturing time, so it is important for a sampling approach to achieve high precision for a fixed number of inspection points. This study designs an efficient adaptive sampling method for the non-uniform rational basis spline (NURBS) curves and surfaces based on deviation analysis. For the free-form curves, it is an iterative method that is used to remove points that are less significant to the reconstruction error from the dense points on the curve. That is, the points are ranked by their maximum deviation from the theoretical curves. Different from the existing methods, a closed-form is derived to approximate the maximum deviation by analyzing the curve reconstruction method, i.e., piecewise cubic spline interpolation. The proposed method is compared with recent curve sampling methods, and the comparison results have shown that, under the same number of inspection points, the reconstruction error of the proposed method is reduced by $82 \%$. The proposed curve sampling algorithm is then further extended to surface sampling, where the global characteristics of a surface are extracted as a series of curves on the surface. Thus, surface sampling is simplified to curve sampling in two directions. The proposed surface sampling strategy is compared with classic surface sampling methods using three representative surfaces. The results show that by using the proposed surface sampling strategy, the reconstruction error is reduced significantly. By applying our sampling method to the on-machine inspection system, the inspection accuracy can be greatly improved.
\end{abstract}

Keywords On-machine inspection• NURBS• Piecewise cubic spline interpolation• Adaptive sampling 


\section{Introduction}

On-machine inspection (OMI) has been widely used in manufacturing high-precision free-form (e.g., turbine blade) or thin-wall surfaces (aerospace structures) [1]. The OMI process requires a certain manufacturing time. The main objective of this study is to achieve high inspection accuracy under a fixed number of inspection points, which is challenging due to the free-form surfaces' complexity and variability. The OMI represents a contact inspection technique, where discrete points are measured precisely by a contact probe and then used to reconstruct a substitute geometry of the corresponding inspected surface. The fabrication error can then be calculated by comparing the substitute geometry and the CAD model. Generally, the more points are measured on the surface, the better reconstruction accuracy will be obtained, but more time will be required for the inspection process. Therefore, developing a suitable sampling strategy for inspection points that requires a fixed number of inspection points while minimizing the reconstruction error is a vital task in the field of OMI measurement.

In recent years, much effort has been devoted to the development of sampling methods. These methods can be roughly divided into two categories: blind sampling methods and adaptive sampling methods. Blind sampling methods use a fixed pattern without considering features such as shape and complexity of geometry. These methods are easy to understand and implement and include various methods, such as uniform sampling of parametric value, arclength, or surface area [2-4]. To increase the discrepancy between points, Hammersley sampling and Halton sampling have been proposed [5,6]. The common disadvantage of blind sampling methods is that much redundant data are acquired, usually implemented in situations where accuracy requirements are not high. Adaptive sampling methods are more intelligent than blind sampling methods. They consider geometrical characteristics and combine optimization methods with point selection to reduce the amount of redundant data to improve inspection efficiency. Adaptive sampling methods can be divided into geometry-guided sampling methods, error-guided sampling methods, and hybrid sampling methods. The geometry-guided sampling methods usually analyze geometrical features, such as knot vectors, curvature, arclength, and inflection points, and combine multiple factors by conferring different weights. These methods enable the inspection points to reach the characteristic distribution [2, 3, 7-10]. The error-guided sampling methods analyze the machine error, reconstruction error, reconstruction uncertainty, and other errors and utilize the error analysis results to determine critical points. These methods typically first generate the initial point set by a simple method and then select the next-best point from the target curve or surface iteratively; this process continues until the desired sampling scale or inspection performance is achieved [11-15]. The hybrid sampling methods use optimization methods or other strategies, such as genetic algorithm, particle swarm optimization algorithm, ant colony algorithm, and bending moment balance theory, to obtain inspection points. The evaluation metric has been commonly used as an objective function to obtain the target inspection points by optimizing the objective function [16-18]. The adaptive sampling methods have made great progress compared with the blind sampling methods. Nevertheless, owing to the complexity of the free-form curve and surface, these methods do not analyze the geometry analytically. Therefore, the higher the reconstruction accuracy they achieve, the greater their time consumption will be.

In this article, an efficient adaptive sampling method for free-form curves and surfaces based on deviation analysis of piecewise cubic spline interpolation is proposed. Also, an iterative point-remove sampling strategy is designed for curve sampling, where the initial points are first obtained by a uniform sampling method in the curve's parameter space and then ranked by their maximum deviation and the least-significant point is removed in every iteration. In addition, a closed-form expression is derived to 
approximate the maximum deviation, which can be computed in an extremely fast way. A comprehensive comparison of the proposed method and several latest sampling methods is performed on several curves. The comparison results show that the reconstruction error can be greatly reduced by the proposed method, i.e., from $0.0422 \mathrm{~mm}$ that is the best classic method result to $0.0082 \mathrm{~mm}$. The proposed curve sampling method is then extended to surface sampling. A non-uniform rational basis spline (NURBS) surface is converted into a tensor product surface. Namely, the global characteristics of a surface are obtained by extracting a series of curves on the surface; thus, the surface sampling is simplified to curve sampling in two directions. The proposed surface sampling strategy is compared with the classic sampling methods, and the results have shown the reconstruction error can be greatly reduced by the proposed strategy.

The rest of this article is organized as follows. In Section 0, two proposed sampling methods for curves and several derivative methods are presented. In Section 0, the proposed curve sampling method is extended to surface sampling. Finally, the main conclusions and future work directions are given in Section 0.

\section{Curve sampling}

The NURBS modeling has been the most popular method for an object's contours presenting in commercial CAD software. A brief introduction to the NURBS is given in the following.

Consider an incremental sequence of knots $\boldsymbol{U}=\left(u_{i}\right)_{i=0}^{n+d}$, where $n$ denotes the number of control points, and $d$ is the degree of this curve. Then, the base function of the NURBS is defined as follows:

$$
\begin{gathered}
B_{i, d}(u)=\frac{u-u_{i}}{u_{i+d}-u_{i}} B_{i, d-1}(u)+\frac{u_{i+d}-u}{u_{i+d+1}-u_{i+1}} B_{i+1, d-1}(u), \\
B_{i, 0}(u)=\left\{\begin{array}{c}
1 \quad \text { if } u_{i} \leq u<u_{i+1} \\
0 \quad \text { otherwise }
\end{array} .\right.
\end{gathered}
$$

Any points on the NURBS curve can be calculated using the corresponding parameter $u$ as follows:

$$
\boldsymbol{C}(u)=\frac{\sum_{i=0}^{n} w_{i} B_{i, d}(u) \boldsymbol{P}_{i}}{\sum_{i=0}^{n} w_{i} B_{i, d}(u)} \quad u_{1} \leq u \leq u_{n+d+1} .
$$

where $\boldsymbol{P}_{i}=\left(x_{i}, y_{i}\right)$ denotes the $i$-th control point, and $w_{i}$ is the weight of the $i$-th control point.

\subsection{Iterative sampling method based on reconstruction deviation}

The iterative sampling based on reconstruction deviation (ISD) is proposed to obtain the minimum inspection points while achieving high reconstruction accuracy. Owing to its high accuracy, its results are used as a reference for the other methods analyzed in this study. Therefore, the ISD algorithm is selected as a baseline and improved to achieve a balance between the accuracy and computational cost.

The main idea of the ISD is to sample points densely on the target inspection curve by a preliminary sampling method, uniform sampling in parametric space (USP) [3] is selected as the preliminary sampling method here. The USP is the simplest sampling strategy that makes the sample points distribute uniformly in the parameter space. These points are referred to as initial points, and they constitute the initial point set denoted as $P$. The ISD removes the less significant points from the curve iteratively.

To select a proper less significant point in every iteration, the feature value $d_{i}$ is introduced to describe the importance of the on-curve point $\boldsymbol{p}_{i}$. As shown in Fig. 1, the feature value denotes the maximum deviation between the substitute geometry and theoretical curve. The substitute geometry is reconstructed by all on-curve sample points except point $\boldsymbol{p}_{i}$, and the feature value of $\boldsymbol{p}_{i}$ can be obtained as follows: 


$$
d_{i}=\max _{u_{1, \min } \leq u_{1} \leq u_{1, \max }}\left[\min _{u_{2, \min } \leq u_{2} \leq u_{2, \max }}\left\|\boldsymbol{C}_{\text {theo }}\left(u_{1}\right)-\boldsymbol{C}_{\text {subs }}\left(u_{2}\right)\right\|\right] .
$$

The feature value of a point denotes the reconstruction error after removing that point. Obviously, the larger the feature value is, the greater the contribution of point removal to the reconstruction error reduction will be. Hence, it is reasonable to use the feature value to describe the importance of a point.

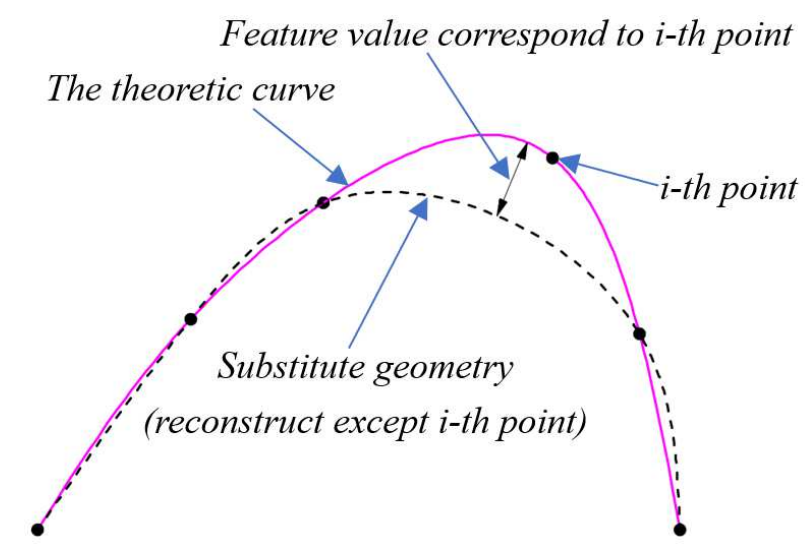

Fig. 1 Definition of the feature value of the $i$ th point

The pseudocode of the ISD is given in Algorithm 1.

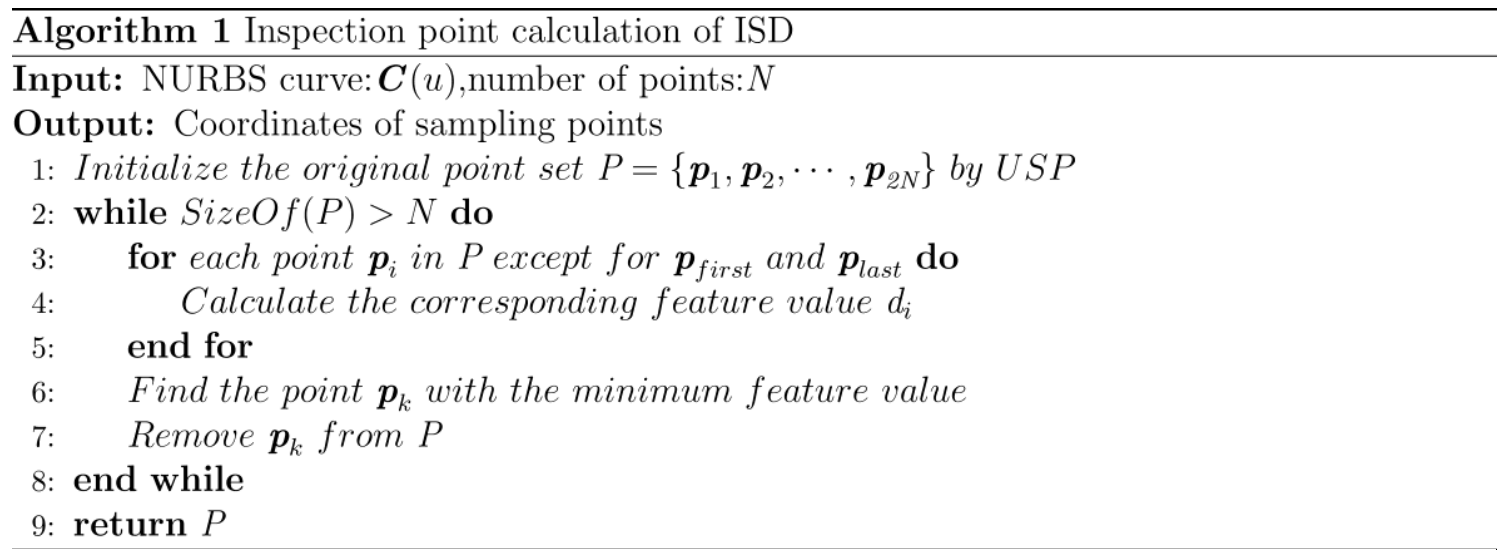

The core of the ISD is to remove the least-important point from the on-curve point set in every iteration. The specific steps are detailed in Algorithm 1. Based on the current on-curve sample points, the ISD first computes the feature values of all the points except two endpoints of the curve and then removes the point with the smallest feature value. This process is repeated until the number of on-curve points meets the predefined requirement. Thus, one of the least important points is removed in every iteration, and the remaining points are essential for curve reconstruction.

In Fig. 2, the on-curve inspection points and their reconstruction errors obtained by the ISD and USP are compared. As shown in Fig. 2, the curve obtained by the ISD is much more accurate than that obtained by the USP; the maximum deviations of the ISD and USP are $0.01139 \mathrm{~mm}$ and $0.04635 \mathrm{~mm}$, respectively. However, the computational time of the ISD is approximately $600 \mathrm{~s}$, and that of the UPS is less than $0.05 \mathrm{~s}$. Thus, the main limitation of the ISD is high time consumption. Fortunately, this shortcoming can be overcome by enhancing the ISD, which will be explained in Section 1.2. 


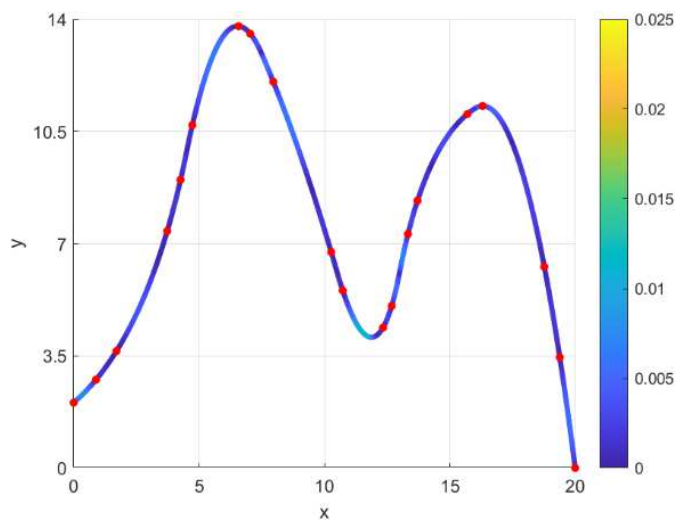

(a) Baseline method ISD

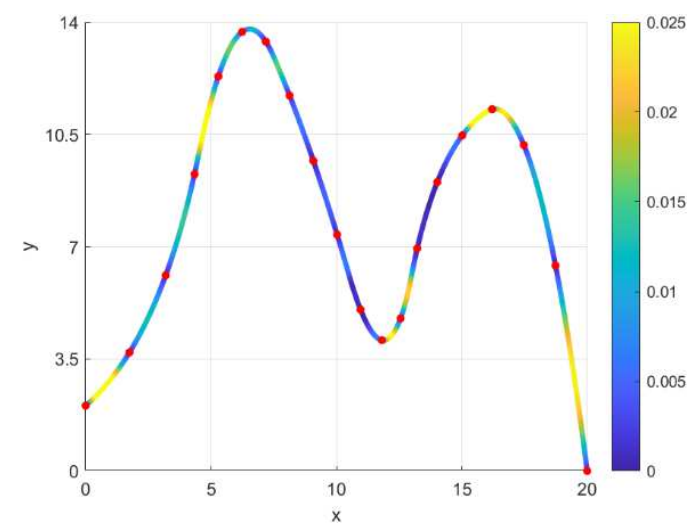

(b) USP

Fig. 2 Reconstruction errors of the ISD and USP for 20 points

\subsection{ISD enhancement}

The ISD could be an effective sampling strategy if its time consumption is reduced. It should be noted that the high time consumption of the ISD is mostly caused by the feature value calculation process. Feature value denotes the maximum deviation between two curves, as given by Eq. (4). However, solving Eq. (4) is a complex nonlinear problem. Thus, it is worth approximating the feature value by a substitute feature value, which can be computed efficiently.

In this study, the piecewise cubic spline interpolation is selected as the default curve reconstruction algorithm, which can guarantee global $\mathcal{C}^{2}$ continuity on a curve. The characteristics of the default curve reconstruction method are analyzed to design an efficient feature value computation algorithm. In this representation, when a sample point is removed, the area closest to it is the most affected one. Therefore, when judging whether the $i$-th point $\boldsymbol{p}_{i}$ should be removed or not, its closest four points should be evaluated locally. Namely, if the two curves reconstructed from the four closest points denoted as $\boldsymbol{p}_{i-2}$, $\boldsymbol{p}_{i-1}, \boldsymbol{p}_{i+1}, \boldsymbol{p}_{i+2}$ and the five closest points $\boldsymbol{p}_{i-2}, \boldsymbol{p}_{i-1}, \boldsymbol{p}_{i}, \boldsymbol{p}_{i+1}, \boldsymbol{p}_{i+2}$ are almost identical, then the point $\boldsymbol{p}_{i}$ can be removed, as shown in Fig. 3(b); otherwise, the point is significant to the curve, as shown in Fig. 3(a).

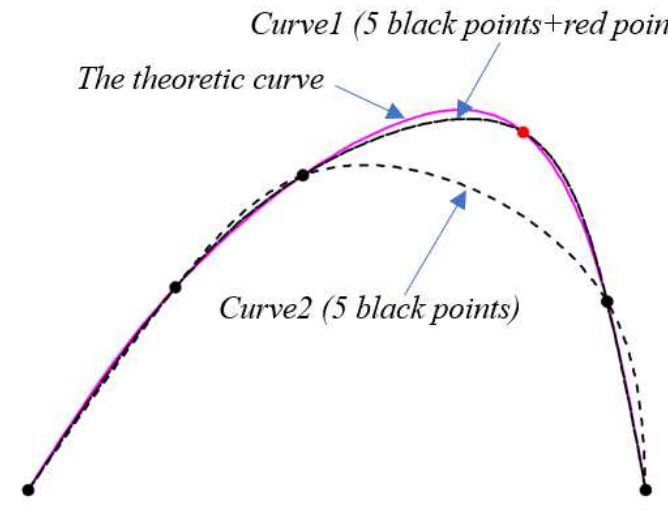

(a) The significant point: red point
Curve1 (5 black points + green point)

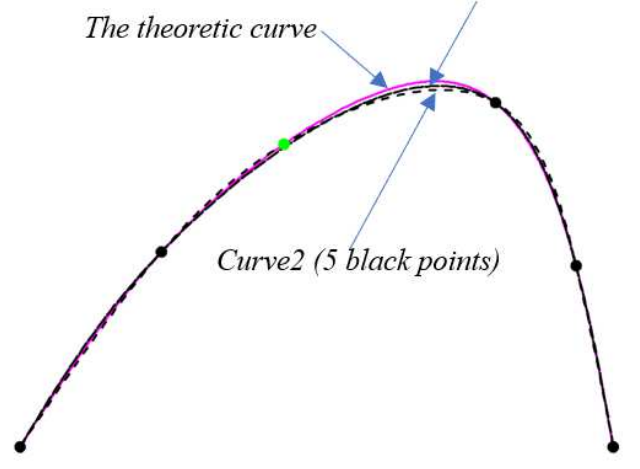

(b) The less significant point: green point

Fig. 3 Illustration of sample point that are less significant and significant to the curve

\subsubsection{Substitute feature value calculation}

Based on the deviation analysis of piecewise cubic spline interpolation, an algorithm for calculating the substitute feature value is proposed. Assume the NURBS curves is expressed as $\boldsymbol{C}(u)=\left\{\begin{array}{l}x=x(u) \\ y=y(u)\end{array}\right.$, 
where $u$ is the coordinate in parametric space, and points on the curve constitute a point set $P=$ $\left\{\boldsymbol{p}_{1}, \boldsymbol{p}_{2}, \cdots, \boldsymbol{p}_{M}\right\}, M>4$. The point set $P$ in the parameter space is expressed as $U=\left\{u_{1}, u_{2}, \cdots, u_{M}\right\}, M>$ $4, u_{1} \leq u_{2} \leq \cdots \leq u_{M}$. Then, the substitute feature value corresponding to point $p_{i}$ is calculated as follows:

(a) For an arbitrary point $\boldsymbol{p}_{i}$ on the curve, its nearest four points $\boldsymbol{p}_{i-2}, \boldsymbol{p}_{i-1}, \boldsymbol{p}_{i+1}$, and $\boldsymbol{p}_{i+2}$ are determined, and the first curve is reconstructed using $\boldsymbol{p}_{i-2}, \boldsymbol{p}_{i-1}, \boldsymbol{p}_{\boldsymbol{i}}, \boldsymbol{p}_{i+1}$, and $\boldsymbol{p}_{i+2}$. The local reconstruction algorithm is a piecewise cubic spline interpolation; it should be noted that take the second boundary condition is required. For a parametric curve, the second derivative at parameter value $u$ can be calculated by

$$
\frac{d^{2} y}{d x^{2}}=\frac{d \frac{d y}{d x}}{d x}=\frac{d \frac{d y / d u}{d x / d u} / d u}{d x / d u}=\frac{\frac{\frac{d^{2} y}{d u^{2}} \cdot \frac{d x}{d u}-\frac{d y}{d u} \cdot \frac{d^{2} x}{d u^{2}}}{(d x / d u)^{2}}}{d x / d u}=\frac{y^{\prime \prime}(u) x^{\prime}(u)-y^{\prime}(u) x^{\prime \prime}(u)}{\left(x^{\prime}(u)\right)^{3}}
$$

Therefore the second boundary condition is defined by $M_{0}=\frac{y^{\prime \prime}\left(u_{i-2}\right) x^{\prime}\left(u_{i-2}\right)-y^{\prime}\left(u_{i-2}\right) x^{\prime \prime}\left(u_{i-2}\right)}{\left(x^{\prime}\left(u_{i-2}\right)\right)^{3}}$ and $M_{4}=\frac{y^{\prime \prime}\left(u_{i+2}\right) x^{\prime}\left(u_{i+2}\right)-y^{\prime}\left(u_{i+2}\right) x^{\prime \prime}\left(u_{i+2}\right)}{\left(x^{\prime}\left(u_{i+2}\right)\right)^{3}}$. The function of the curve reconstructed by the piecewise cubic spline interpolation is a cubic polynomial at every interval, and it can be reorganized as follows:

$$
s_{1}(x)=\left\{\begin{array}{c}
A_{0} x^{3}+B_{0} x^{2}+C_{0} x+D_{0}, x \in\left[x_{i-2}, x_{i-1}\right] \\
A_{1} x^{3}+B_{1} x^{2}+C_{1} x+D_{1}, x \in\left[x_{i-1}, x_{i}\right] \\
A_{2} x^{3}+B_{2} x^{2}+C_{2} x+D_{2}, x \in\left[x_{i}, x_{i+1}\right] \\
A_{3} x^{3}+B_{3} x^{2}+C_{3} x+D_{3}, x \in\left[x_{i+1}, x_{i+2}\right]
\end{array} .\right.
$$

Similarly, the second curve is reconstructed using $\boldsymbol{p}_{i-2}, \boldsymbol{p}_{i-1}, \boldsymbol{p}_{i+1}$, and $\boldsymbol{p}_{i+2}$. The second boundary condition is defined by $M_{0}=\frac{y^{\prime \prime}\left(u_{i-2}\right) x^{\prime}\left(u_{i-2}\right)-y^{\prime}\left(u_{i-2}\right) x^{\prime \prime}\left(u_{i-2}\right)}{\left(x^{\prime}\left(u_{i-2}\right)\right)^{3}}$ and $M_{3}=$ $\frac{y^{\prime \prime}\left(u_{i+2}\right) x^{\prime}\left(u_{i+2}\right)-y^{\prime}\left(u_{i+2}\right) x^{\prime \prime}\left(u_{i+2}\right)}{\left(x^{\prime}\left(u_{i+2}\right)\right)^{3}}$ and can be reorganized as follows:

$$
s_{2}(x)=\left\{\begin{array}{l}
E_{0} x^{3}+F_{0} x^{2}+G_{0} x+H_{0}, x \in\left[x_{i-2}, x_{i-1}\right] \\
E_{1} x^{3}+F_{1} x^{2}+G_{1} x+H_{1}, x \in\left[x_{i-1}, x_{i+1}\right] \\
E_{2} x^{3}+F_{2} x^{2}+G_{2} x+H_{2}, x \in\left[x_{i+1}, x_{i+2}\right]
\end{array}\right.
$$

The illustrations of $s_{1}(x)$ and $s_{2}(x)$ is shown as Fig. 4.

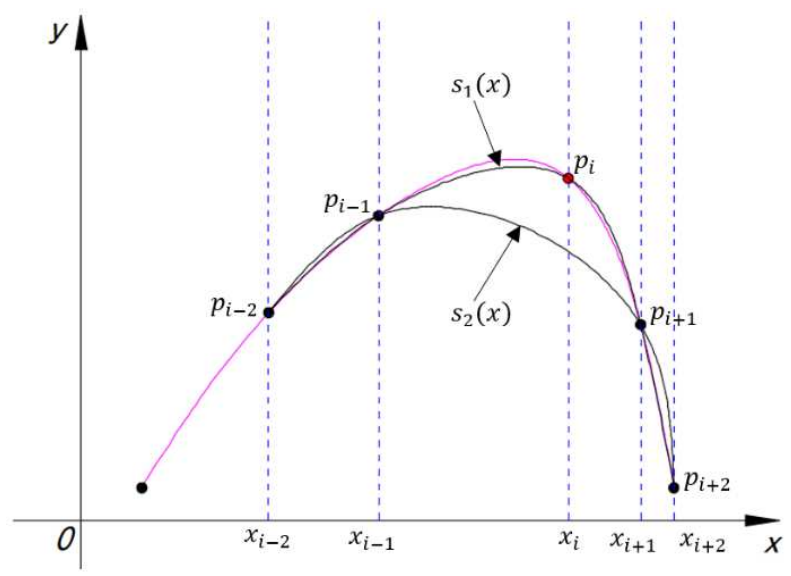

Fig. 4 Illustration of $s_{1}(x)$ and $s_{2}(x)$. The magenta curve denotes the theoretic curve 
(b) When $x \in\left[x_{i-2}, x_{i-1}\right]$, it is possible to compute the distance between $s_{1}(x)$ and $s_{2}(x)$ in the direction of the $y$-axis as follows:

$$
|\Delta y|=\left|s_{1}(x)-s_{2}(x)\right|=\left|\left(A_{0}-E_{0}\right) x^{3}+\left(B_{0}-F_{0}\right) x^{2}+\left(C_{0}-G_{0}\right) x+\left(D_{0}-H_{0}\right)\right| .
$$

It should be noted that the dependent variable $\Delta y$ is a cubic polynomial and equals zero at the two end points of $\left[x_{i-2}, x_{i-1}\right]$; thus, $\frac{d \Delta y}{d x}=0$ must be satisfied when the maximum value of $|\Delta y|$ is obtained in the interval of $\left[x_{i-2}, x_{i-1}\right]$, and the value of $\frac{d \Delta y}{d x}$ can be calculated by

$$
\frac{d \Delta y}{d x}=3\left(A_{0}-E_{0}\right) x^{2}+2\left(B_{0}-F_{0}\right) x+\left(C_{0}-G_{0}\right)
$$

Two solutions to $\frac{d \Delta y}{d x}=0$ are calculated by

$$
\begin{aligned}
& x_{0}=\frac{-\left(B_{0}-F_{0}\right)+\sqrt{\left(B_{0}-F_{0}\right)^{2}-3\left(A_{0}-E_{0}\right)\left(C_{0}-G_{0}\right)}}{3\left(A_{0}-E_{0}\right)}, \\
& x_{1}=\frac{-\left(B_{0}-F_{0}\right)-\sqrt{\left(B_{0}-F_{0}\right)^{2}-3\left(A_{0}-E_{0}\right)\left(C_{0}-G_{0}\right)}}{3\left(A_{0}-E_{0}\right)} .
\end{aligned}
$$

If $x_{0}$ is a real number in the interval of $\left[x_{i-2}, x_{i-1}\right]$, it is added to the dataset $X_{\text {aim }}$; otherwise, it is discarded. The same process is repeated for solution $x_{1}$.

(c) When $x \in\left[x_{i-1}, x_{i}\right]$, the deviation between $s_{1}(x)$ and $s_{2}(x)$ in the direction of the $y$-axis is calculated by

$$
|\Delta y|=\left|\left(A_{1}-E_{1}\right) x^{3}+\left(B_{1}-F_{1}\right) x^{2}+\left(C_{1}-G_{1}\right) x+\left(D_{1}-H_{1}\right)\right| .
$$

Situation in this interval is different from that in the interval of $\left[x_{i-2}, x_{i-1}\right]$. Namely, the value of $|\Delta y|$ is not equal to zero at an end point of $\left[x_{i-1}, x_{i}\right]$, and under this circumstance, the maximum value of $|\Delta y|$ may be obtained not only at $\frac{d \Delta y}{d x}=0$ but also at an endpoint of $\left[x_{i-1}, x_{i}\right]$. However, the processing is the same as in the previous step, and generated vulnerability will be counteracted later.

By solving the equation of $\frac{d \Delta y}{d x}=0$ in the interval of $\left[x_{i-1}, x_{i}\right]$, two solutions are obtained as follows:

$$
\begin{aligned}
& x_{2}=\frac{-\left(B_{1}-F_{1}\right)+\sqrt{\left(B_{1}-F_{1}\right)^{2}-3\left(A_{1}-E_{1}\right)\left(C_{1}-G_{1}\right)}}{3\left(A_{1}-E_{1}\right)}, \\
& x_{3}=\frac{-\left(B_{1}-F_{1}\right)-\sqrt{\left(B_{1}-F_{1}\right)^{2}-3\left(A_{1}-E_{1}\right)\left(C_{1}-G_{1}\right)}}{3\left(A_{1}-E_{1}\right)} .
\end{aligned}
$$

Solutions $x_{2}$ and $x_{3}$ are handled in the same way as solution $x_{0}$.

(d) In the case of $x \in\left[x_{i}, x_{i+1}\right]$ and $x \in\left[x_{i+1}, x_{i+2}\right]$, the processing is the same as for $x \in\left[x_{i-1}, x_{i}\right]$ and $\left[x_{i-2}, x_{i-1}\right]$, respectively. To compensate the vulnerability left from the previous step, $x_{i}$ will be added to the dataset $X_{\text {aim }}$ at the end of this procedure.

(e) For each value in dataset $X_{\text {aim }}$, the corresponding $|\Delta y|$ is calculated; the expression of $|\Delta y|$ varies from the independent variables range; the value of $|\Delta y|$ is added to dataset $Y_{\text {aim }}=\{\mid \Delta y(x) \| x \in$ $\left.X_{\text {aim }}\right\}$. The geometric significance of $|\Delta y|$ is shown in Fig. 5. 


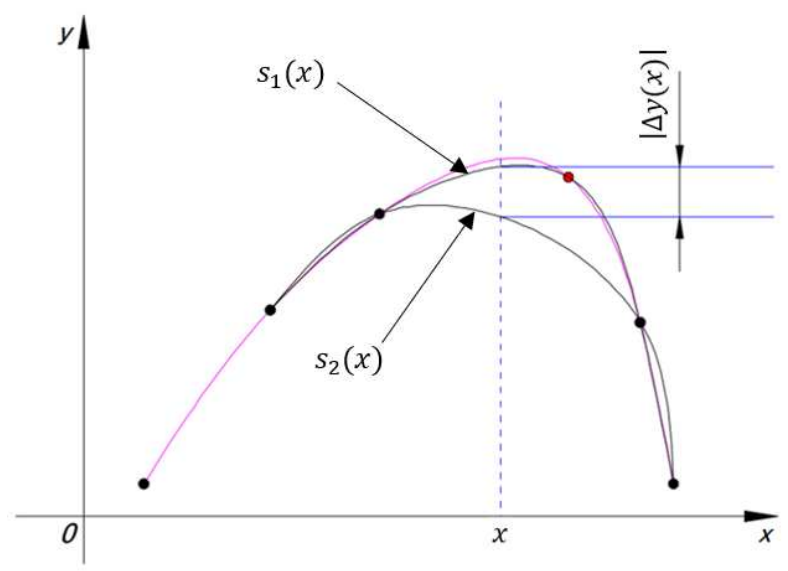

Fig. 5 Geometrical significance of $|\Delta y|$

(f) The suspicious values of maximum $|\Delta y|$ have been stored in the dataset $Y_{\text {aim }}$. The maximum deviation between $s_{1}(x)$ and $s_{2}(x)$, which is expressed as $d=\max _{x_{i-2} \leq x_{1} \leq x_{i+2}}\left[\min _{x_{i-2} \leq x_{2} \leq x_{i+2}} \sqrt{\left(x_{1}-x_{2}\right)^{2}+\left(s_{1}\left(x_{1}\right)-s_{2}\left(x_{2}\right)\right)^{2}}\right]$ should be calculated, but the analytical solution to this equation is hard to obtain, so in this study, an approximate algorithm is proposed to solve it. Details of the approximate algorithm are shown in Fig. 6.

Assume $x_{a}$ is an arbitrary value in dataset $X_{\text {aim }}$; by substituting $x_{a}$ into Eq. (6) and Eq. (7), two points $A\left(x_{a}, s_{1}\left(x_{a}\right)\right), B\left(x_{a}, s_{2}\left(x_{a}\right)\right)$ will be obtained; pass through the point $A$ to draw the normal and tangent of $s_{1}(x)$, and then pass through the point $B$ to draw the vertical line of the normal, their intersection point is point $C$.

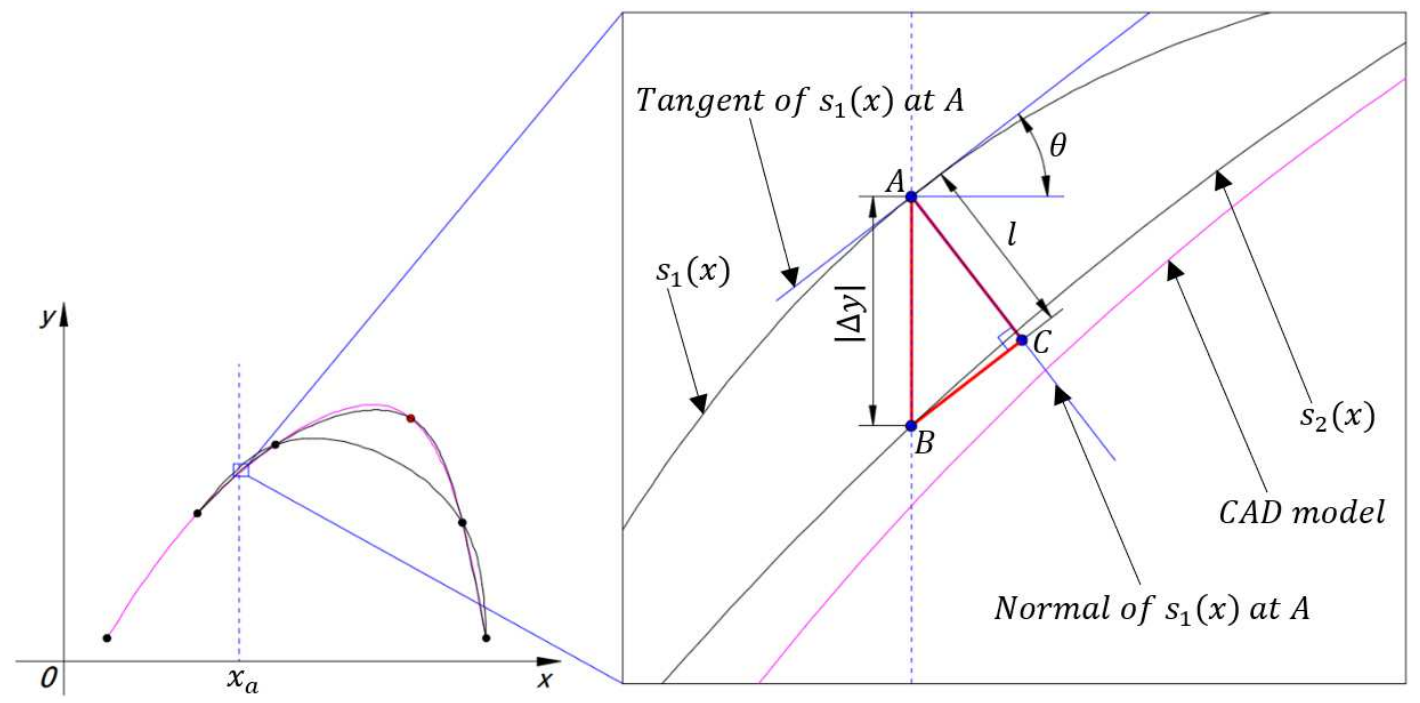

Fig. 6 Schematic of the approximate algorithm

The length of line $A C$ is $l$, which is treated as an approximation of the deviation at $x_{a}$ and is the target in this section; $\theta$ is the tilting angle of the tangent. It should be noted that $l=|\Delta y| \cdot|\cos \theta|$, the value of $|\Delta y|$ is known, and $|\cos \theta|$ could be obtained as follows:

$$
|\cos \theta|=\sqrt{\cos ^{2} \theta}=\sqrt{\frac{\cos ^{2} \theta}{\sin ^{2} \theta+\cos ^{2} \theta}}=\sqrt{\frac{1}{1+\tan ^{2} \theta}} .
$$

The value of $\tan \theta$ is equal to $s_{1}^{\prime}\left(x_{a}\right)$ and can be accessed as 


$$
\tan \theta=s_{1}^{\prime}(x)=\left\{\begin{array}{c}
3 A_{0} x^{2}+2 B_{0} x+C_{0}, x \in\left[x_{i-2}, x_{i-1}\right] \\
3 A_{1} x^{2}+2 B_{1} x+C_{1}, x \in\left[x_{i-1}, x_{i}\right] \\
3 A_{2} x^{2}+2 B_{2} x+C_{2}, x \in\left[x_{i}, x_{i+1}\right] \\
3 A_{3} x^{2}+2 B_{3} x+C_{3}, x \in\left[x_{i+1}, x_{i+2}\right]
\end{array} .\right.
$$

Therefore, $l$ is obtained in this step. For each value in dataset $Y_{\text {aim }}$, the corresponding $l$ is calculated and added to the dataset $L, L=\left\{|\cos \theta| \cdot|\Delta y||| \Delta y \mid \in Y_{\text {aim }}\right\}$.

(g) Finally, the maximum value of dataset $L$ is taken as a substitute feature value corresponding to point $\boldsymbol{p}_{i}$.

\subsubsection{Enhanced ISD}

Based on the above ideas, an enhanced ISD (EISD) is proposed to obtain inspection points. This method calculates the feature points first, and then adds auxiliary points according to the parameter value of on-curve points progressively. For the calculation method of substitute feature value is an approximation algorithm, and auxiliary points are taken to compensate for the cumulative error. The pseudocode of the EISD is given in Algorithm 2.

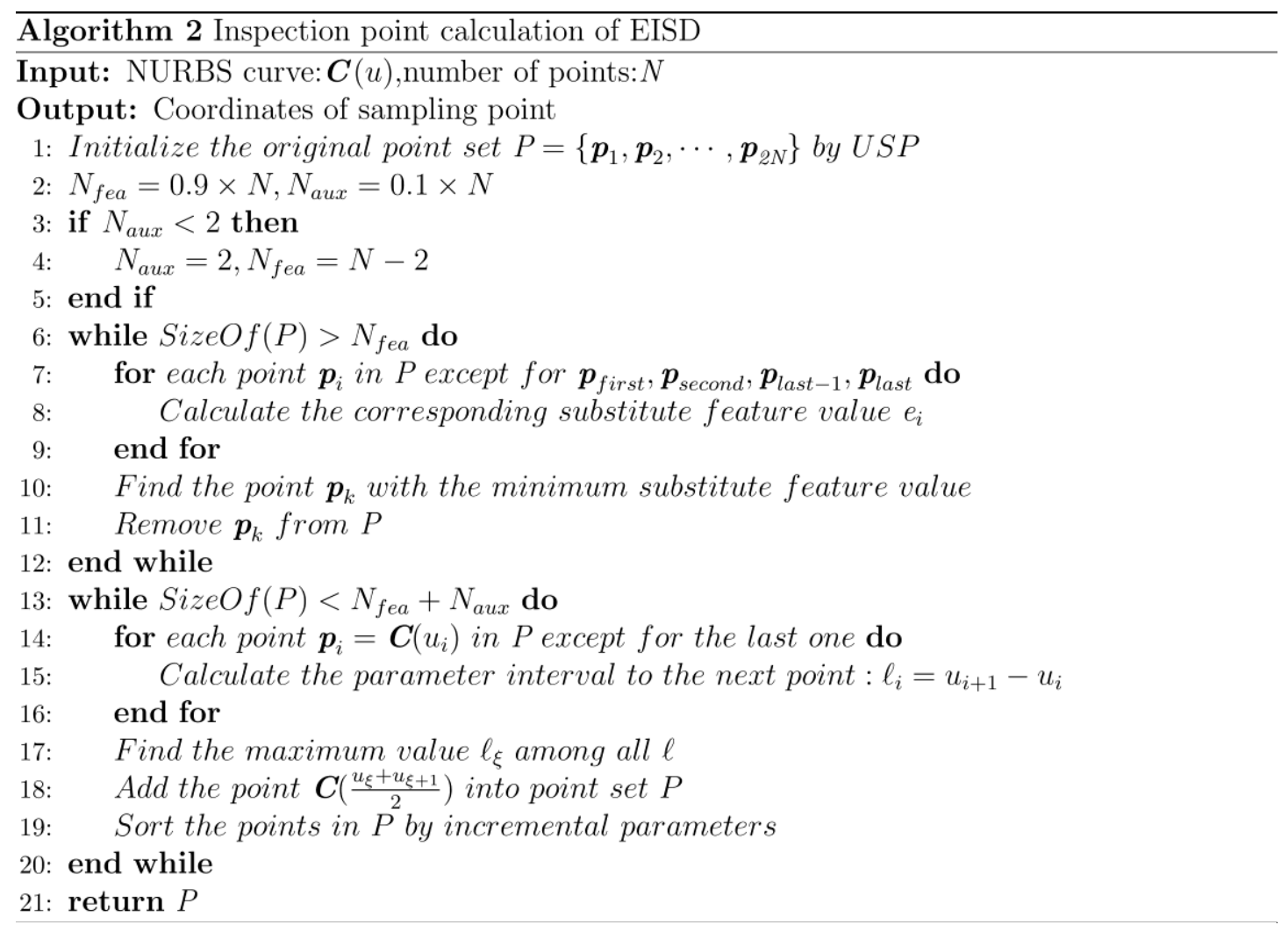

The main difference between the ISD and the EISD is that in the EISD, the substitute feature value is used as a feature value to guide point deletion, and the amend stage is added to the end of the points removal procedure. The EISD algorithm can be mainly divided into two steps. In the first step, the initial point set is constructed, and the feature points and auxiliary points are determined. In the second step, the calculation of the substitute feature value is performed multiple times, so it will which takes up a large proportion of total calculation time. The time for the USP to generate the initial point set is negligible. In this study, the time of computing substitute feature value is regarded as the time complexity of the EISD.

When the capacity of the initial point set is $2 N$, the time complexity is $T(N)=\frac{(2 N+[0.9 N]) \cdot(2 N-[0.9 N]+1)}{2} \approx$ $1.959 N^{2}+1.45 N \approx 2 N^{2}$, where the brackets indicate the rounding function. 


\subsection{Curve sampling experiments}

The piecewise cubic spline interpolation with natural boundary condition is taken as the default reconstruction algorithm. Owing to the limitation of the piecewise cubic spline interpolation, curves used to sample points must satisfy the requirement that there is no overlapping segment when the curve is orthogonally projected onto the $x$-axis; otherwise, the target curve should be split into non-overlap segments.

The maximum deviation between the substitute geometry and the theoretic curve is used as an evaluation metric. This metric is computed as the radius of a maximum tangent circle: the center of the circle is on the theoretic curve, and it is tangent to the substitute geometry, as shown in Fig. 7. It should be noted that the substitute feature value (introduced in Section 1.2.1) is used only to select the less significant points but not to compute the maximum deviation.

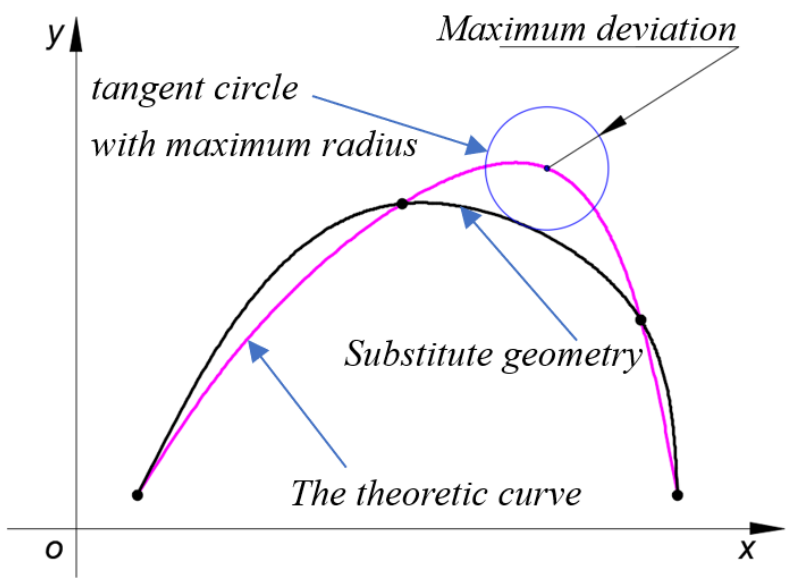

Fig. 7 Schematic of the tangent circle method

\subsubsection{Accuracy study on vital factors}

The main objective is to minimize the reconstruction error under the required number of points. In the EISD, the quantity (i.e., different number of initial points in $P$ ) and quality (different sampling scheme to obtain the initial points) of points in the initial point set $P$ could greatly affect the reconstruction error.

\section{Quantity of initial points}

Suppose $N$ is the number of target inspection points, and $k N$ is the number of initial points, where $k$ is a positive integer. In the experiments, when $k$ refers to the magnification of the EISD, it is denoted as EISD- $k$. In this section, the USP is used to obtain the initial points.

The effect of magnification on reconstruction error is presented in Fig. 8. The selected NURBS curve for experiment is presented in Fig. 12(c), and the maximum deviation and computing time with respect to $k$ magnification are presented in Fig. 8. As shown in Fig. 8, as the magnification increases, the maximum deviation tends to reduce. In addition, the maximum deviation shows no significant difference when the number of inspection points is larger than 30 , and the magnification is greater than 7 . Based on the reconstruction error of the ISD for the number of target inspection points of 20 and 30, the reconstruction accuracy of the EISD-7 exceeds that of the ISD. According to the definition of algorithm complexity, given

in Section 1.2.2, the algorithm complexity of the EISD- $k$ is $T(k, N)=\frac{(k N+[0.9 N]) \cdot(k N-[0.9 N]+1)}{2} \approx$ $\left(0.5 k^{2}-0.405\right) N^{2}+(0.5 k+0.45) N \approx 0.5 k^{2} N^{2}$, which indicates that the computing time increases at a rate of squared $k$, as the magenta curve in Fig. 8 shows. To achieve the trade-off between the reconstruction accuracy and computing time, when the reconstruction error meets the requirements, the magnification 
should be reduced as much as possible. When the magnification is seven, the reconstruction error is reduced a lot compared with EISD, which meets the requirements in most cases, and the calculation process consumes less time, therefore, the magnification by seven is a good choice.

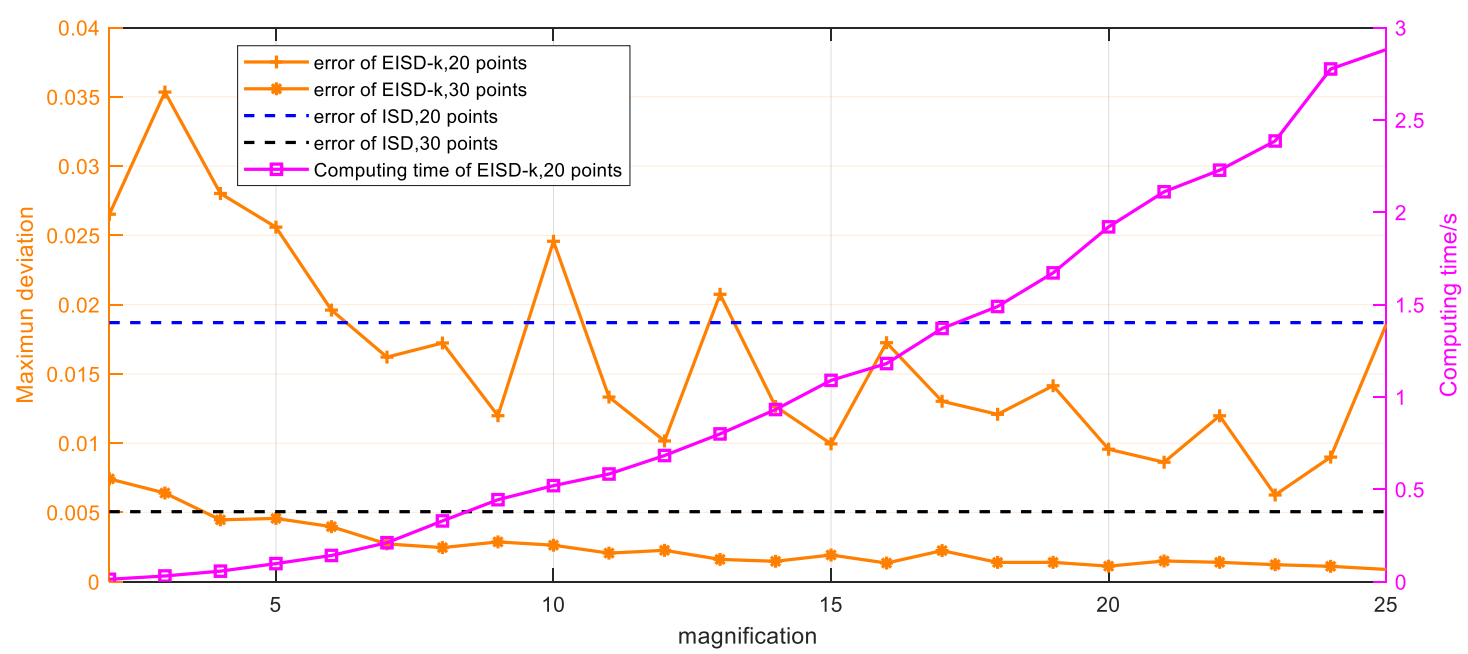

Fig. 8 The effect of magnification

\section{Quality of initial points}

In Section 1.2.2, the initial point set $P$ of the EISD is generated by the USP; however, if $P$ is alternatively sampled by the EISD, a better quality of initial points could be obtained. For the algorithm in Section 1.2.2, we adjust it as follows, the initial point set $P$ is generated by EISD- $k_{l}$ instead of USP, the number of initial points is adjusted to $k_{2}$, and the rest remains unchanged, this derivative method is referred as the EISD-L2 $\left(k_{1}, k_{2}\right)$, where L2 means that the algorithm presented in Section 1.2.2 is performed twice. First, it is used to generate the initial point set $P$, performing the magnification by $k_{1}$. Second, it is used to calculate the final inspection points, performing the magnification by $k_{2}$. By analogy, the meaning of the EISD-L3 $\left(k_{1}, k_{2}, k_{3}\right)$ can be explained. A complex curve shown in Fig. 12(c) is used to study the influence of initial point quality on reconstruction error in this section.

The effects of different magnifications in the EISD-L2 $\left(k_{1}, k_{2}\right)$ and EISD-L3 $\left(k_{1}, k_{2}, k_{3}\right)$ on reconstruction error are presented in Fig. 9 and Fig. 10, respectively. In Fig. 9 and Fig. 10, the color of squares indicates the reconstruction error value. Based on the results presented in Fig. 9 and Fig. 10, the quality of initial points has a significant effect on the reconstruction error. The general tendency is that the more layers and the higher magnification are used, the smaller reconstruction error will be achieved. It should be noted that the graphs in Fig. 9 andFig. 10 are highly symmetrical; hence, $k_{1}$ and $k_{2}$ in the EISD-L2 $\left(k_{1}, k_{2}\right)$ are equivalent, as well as $k_{1}, k_{2}$, and $k_{3}$ in the EISD-L3 $\left(k_{1}, k_{2}, k_{3}\right)$; for instance, the EISD-L2 $(2,3)$ and the EISD-L2 $(3,2)$ will provide the same result.

According to the definition of algorithm complexity in Section 1.2.2, the algorithm complexities of the $\operatorname{EISD}-\mathrm{L} 2\left(k_{1}, k_{2}\right)$ and $\operatorname{EISD-L3}\left(k_{1}, \quad k_{2}, \quad k_{3}\right)$ are $T_{2}\left(k_{1}, k_{2}, N\right)=T\left(k_{1} \times k_{2}, N\right)+T\left(k_{2}, N\right) \approx$ $0.5 k_{1}^{2} k_{2}^{2} N^{2}+0.5 k_{2}^{2} N^{2} \quad$ and $\quad T_{3}\left(k_{1}, k_{2}, k_{3}, N\right)=T_{2}\left(k_{1}, k_{2}, k_{3} \times N\right)+T\left(k_{3}, N\right) \approx 0.5 k_{1}^{2} k_{2}^{2} k_{3}^{2} N^{2}+$ $0.5 k_{2}^{2} k_{3}^{2} N^{2}+0.5 k_{3}^{2} N^{2}$, respectively. Obviously, a larger magnification will result in lower sampling efficiency, as shown in Fig. 11, so the magnification should be as small as possible. It can be seen from Fig. 9 that, compared with EISD-L2(2, 2), the reconstruction error of EISD-L2(3, 3) is reduced a lot, and the magnification is very low. In the case of other higher magnifications, although the reconstruction error will continue to decrease, it is not obvious, and the calculation time will be greatly increased. It can be seen from Fig. 10 that there are similar conclusions for EISD-L3 $\left(k_{1}, k_{2}, k_{3}\right)$, the reconstruction accuracy of 
EISD-L3(3, 3, 3) is relatively high and the magnification is very small. Considering the accuracy and computing time, the EISD-L2(3, 3) and EISD-L3(3, 3, 3) are desirable methods.

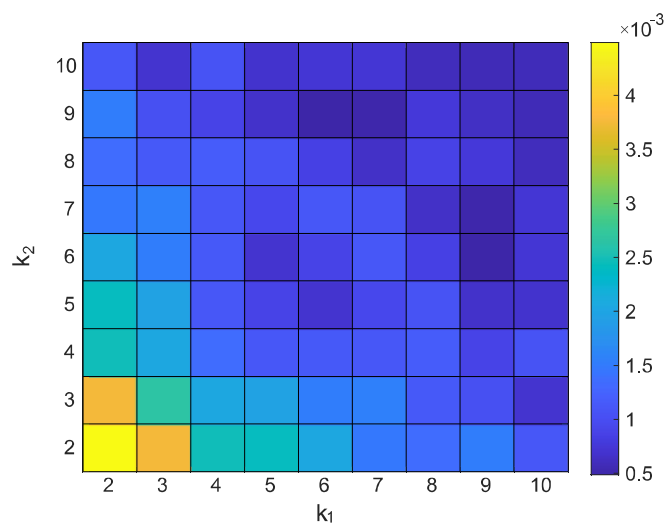

(a) 30 inspection points

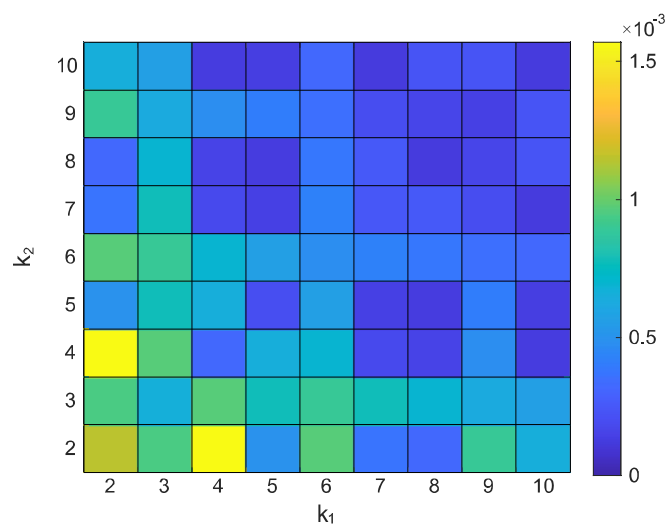

(b) 50 inspection points

Fig. 9 Reconstruction error of the EISD-L2 $\left(k_{1}, k_{2}\right)$

When the initial point set $P$ is obtained by the USP, that is equivalent to the EISD- $k$; the reconstruction result of the EISD- $k$ is shown in Fig. 8, and obviously, the accuracy of EISD-L2 $\left(k_{1}, k_{2}\right)$ is higher than EISD- $k_{2}$.

The reconstruction results of the EISD-L3 $\left(k_{1}, k_{2}, k_{3}\right)$ are shown in Fig. 10, where the color of the squares indicates the reconstruction error value.

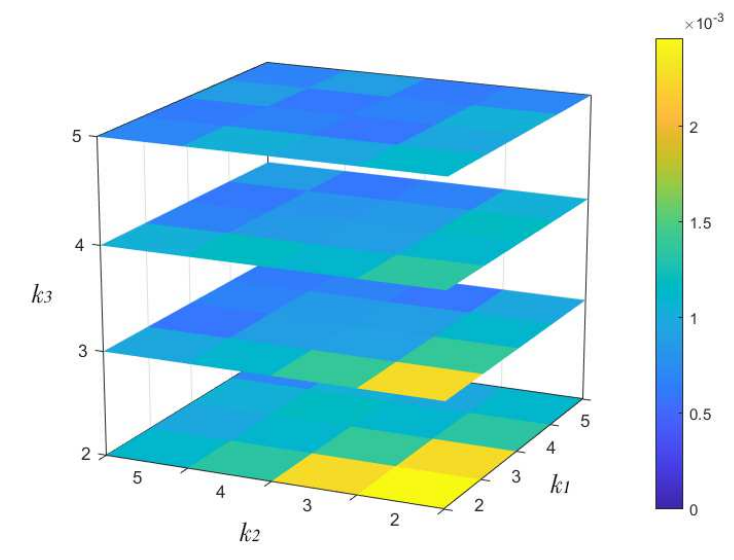

(a) 30 inspection points

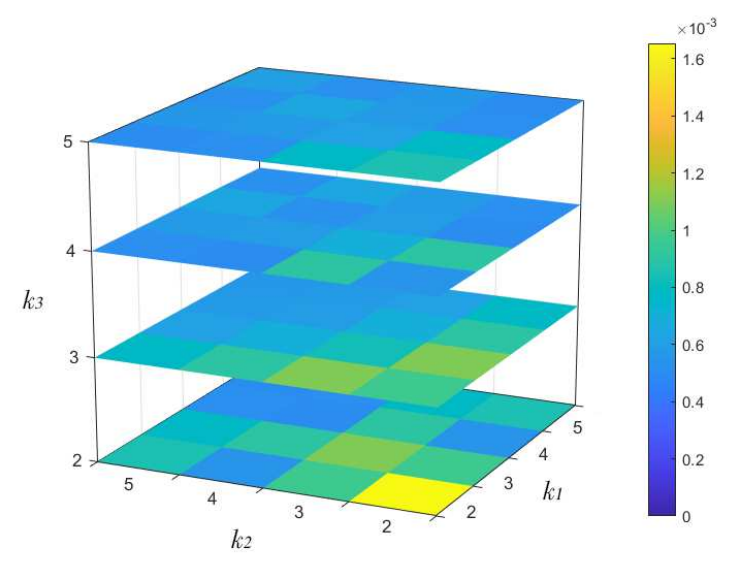

(b) 50 inspection points

Fig. 10 Reconstruction error of the EISD-L3 $\left(k_{1}, k_{2}, k_{3}\right)$

When the initial point set $P$ is obtained by the EISD- $k$, that is equivalent to the EISD-L2 $\left(k_{1}, k_{2}\right)$; the reconstruction result of the EISD-L2 $\left(k_{1}, k_{2}\right)$ is shown in Fig. 10, where it can be seen that improving the quality of initial points is beneficial to improve accuracy. 


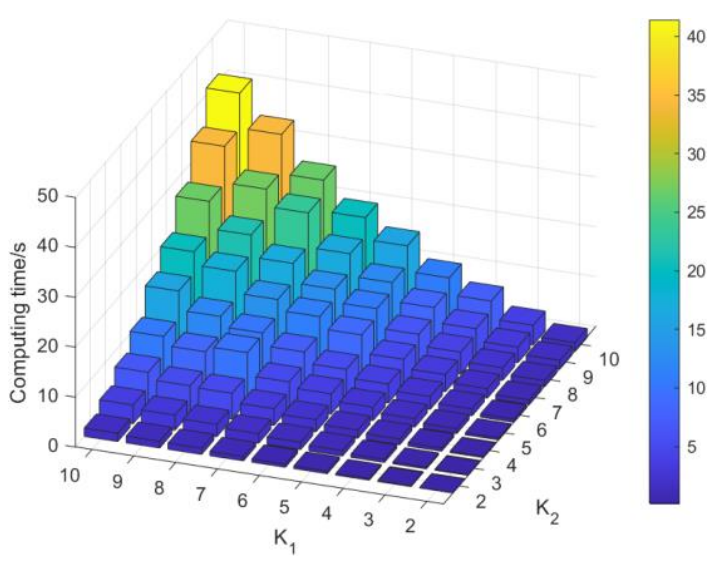

(a) $\operatorname{EISD}-\mathrm{L} 2\left(k_{1}, k_{2}\right)$

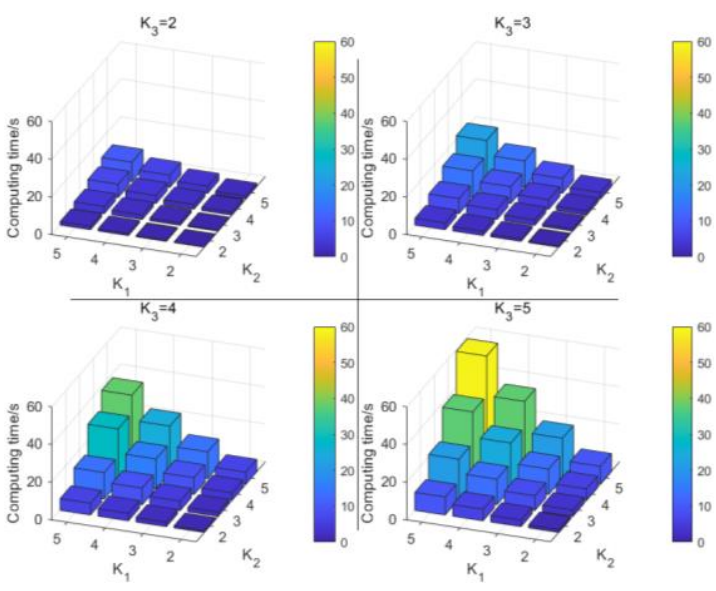

(b) $\operatorname{EISD-L3}\left(k_{1}, k_{2}, k_{3}\right)$

Fig. 11 Computing times of two sampling method for 20 inspection points

\subsubsection{Comparison with other curve sampling methods}

We compare the proposed method with several classical curve sampling methods, these methods are widely used in practice and are very representative.

The curve sampling methods used for comparison are ISD, USP, uniform sampling of arc length (USL) [4], uniform sampling of arc length and accumulative curvature (USLC) [2], EISD-7, EISD-L2(3,3), and EISD-L3(3,3,3). The methods were compared on three NURBS curves having different geometrical complexities. The first one was a simple smooth curve, as shown in Fig. 12(a). The second one was a complex curve, which included different numbers of inflection points and curvature changes, as shown in Fig. 12(c). The third curve contained many sharp corners, as shown in Fig. 12(e). Comparison results are presented in Fig. 12-Fig. 14.

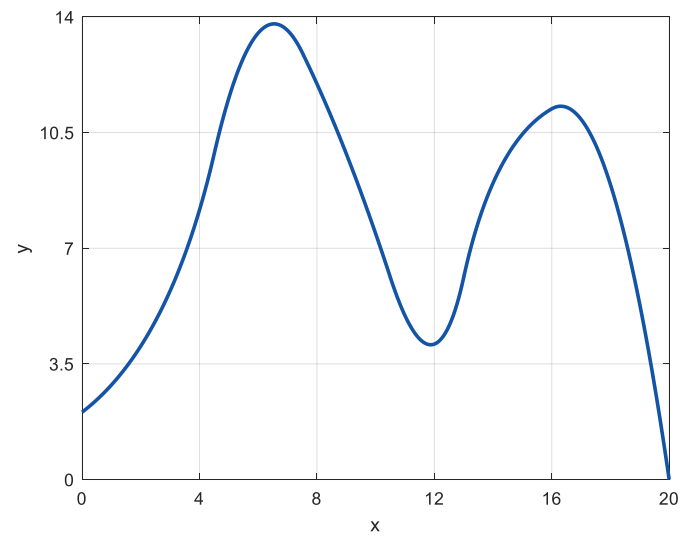

(a) NURBS curve 1

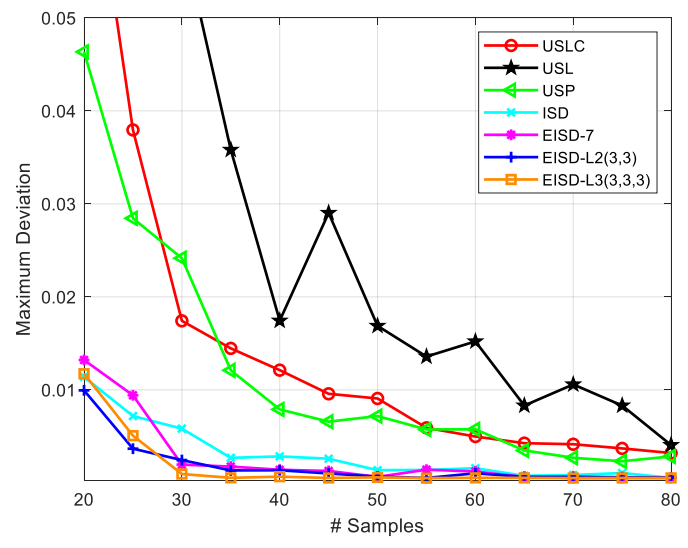

(b) Maximum deviation as a function of sample number 


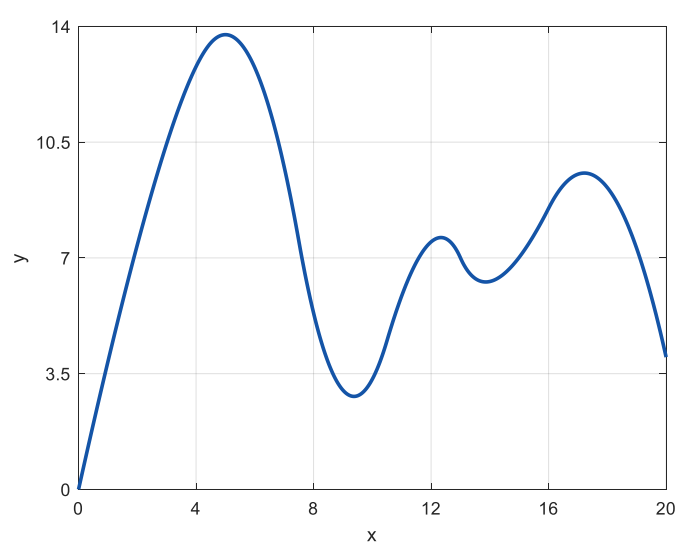

(c) NURBS curve 2

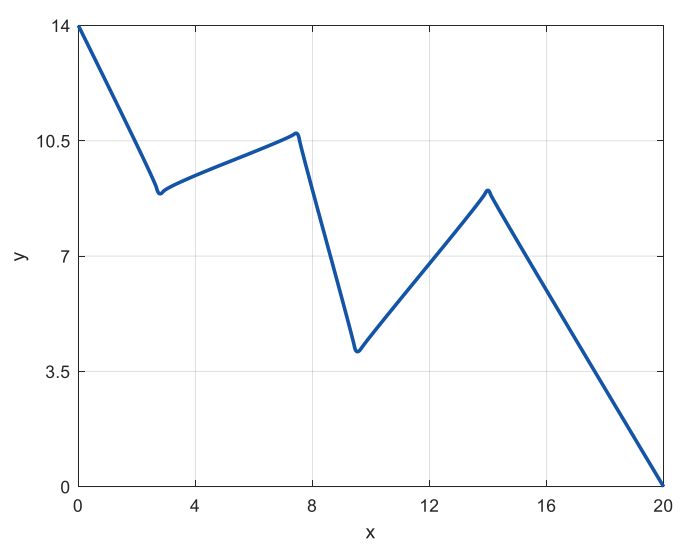

(e) NURBS curve 3

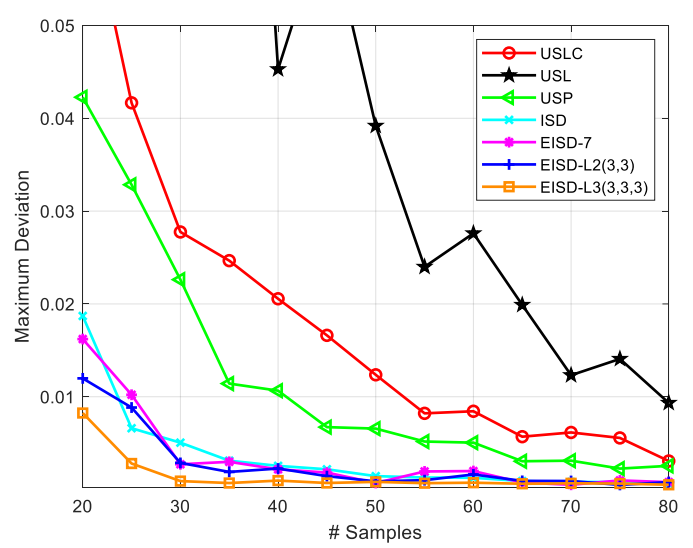

(d) Maximum deviation as a function of sample number

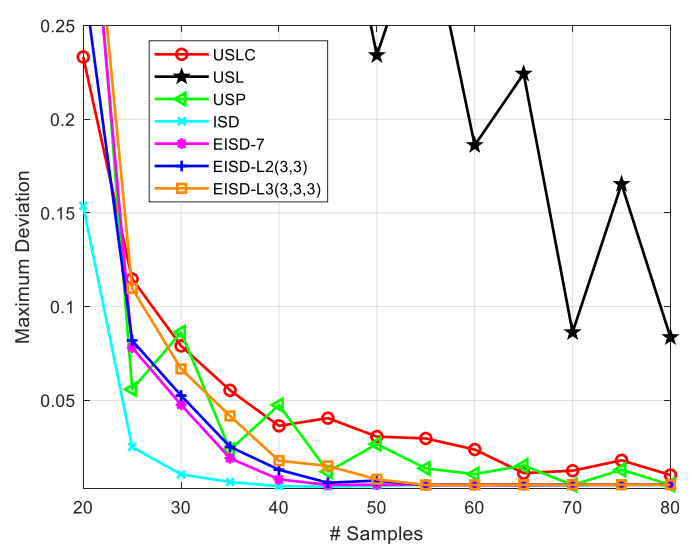

(f) Maximum deviation as a function of sample number

Fig. 12 Comparison of different sampling methods on different NURBS curves

Since the experimental result of the EISD is similar to those presented in Fig. 12, it is omitted in Fig. 12. As shown in Fig. 12(b), 12(d), and 12(f), in most cases, the reconstruction accuracy of the proposed methods exceeded those of the USP, USL, and USLC. According to the polylines shown in Fig. 12(b) and 12(d), the EISD-7, EISD-L2(3,3), and EISD-L3(3,3,3) performed better than the other sampling methods, which has indicated that these methods are effective for curves of various complexity levels and are capable of improving the reconstruction accuracy of the ISD. Nevertheless, as shown in Fig. 12(f), the accuracy of the ISD was highest when the number of inspection points was below 45; therefore, the ISD is suitable for curves that contain sharp corners. The results in Fig. 12 differ from those obtained by Pagani and Scott [2], which could be due to the difference in curve characteristics and performance indicators.

The distribution of critical points along the curve and the reconstruction error of different methods are shown in Fig. 13. As shown in Fig. 13(b) and Fig. 13(c), the EISD-7 and EISD-L3(3,3,3) had better performances than the other sampling strategies, because these algorithms could capture curves' critical points well, including high-curvature points and inflection points. The reconstruction error of the USL was high in the high-curvature segments, as shown in Fig. 13(f). As displayed in Fig. 13(d) and 13(e), the USLC performed worse than the USP; a large reconstruction error occurred at the neighbor area of the inflexion points. The inflection points were ignored by the USLC since the curvature at inflection points equaled zero. As shown in Fig. 13(c), there were two sample points near the inflexion points. Therefore, it can be concluded that inflexion points are critical for curve reconstruction, which is consistent with the conclusion drawn by Lu et al. [9]. 


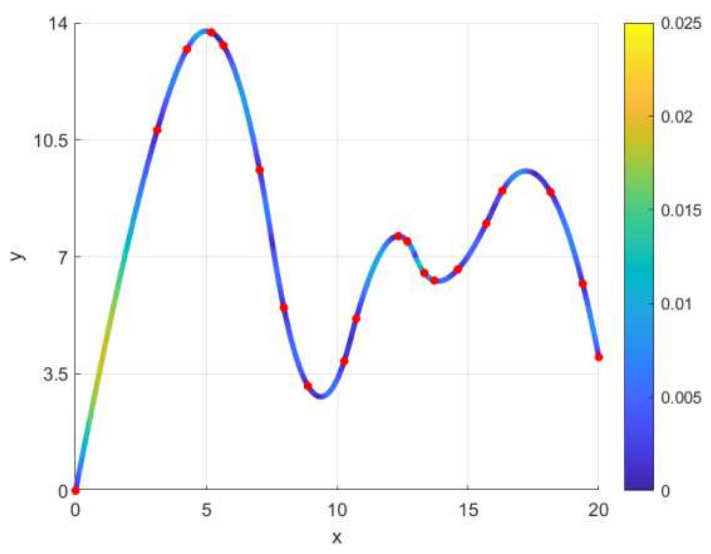

(a) ISD

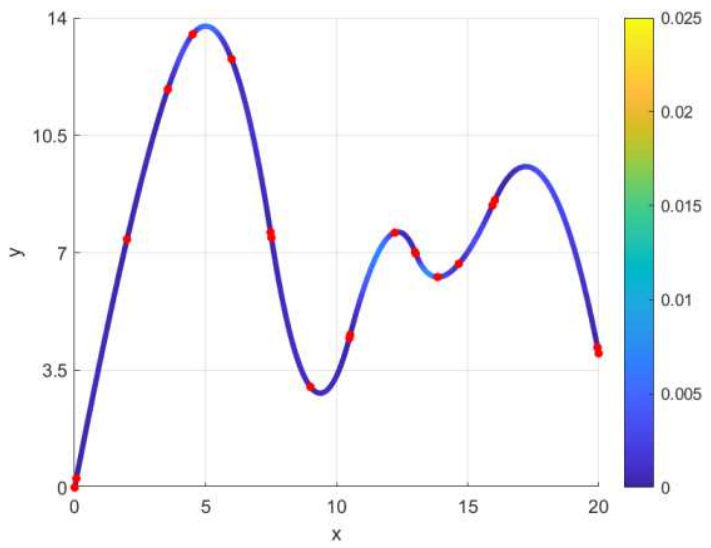

(c) EISD-L3(3,3,3)

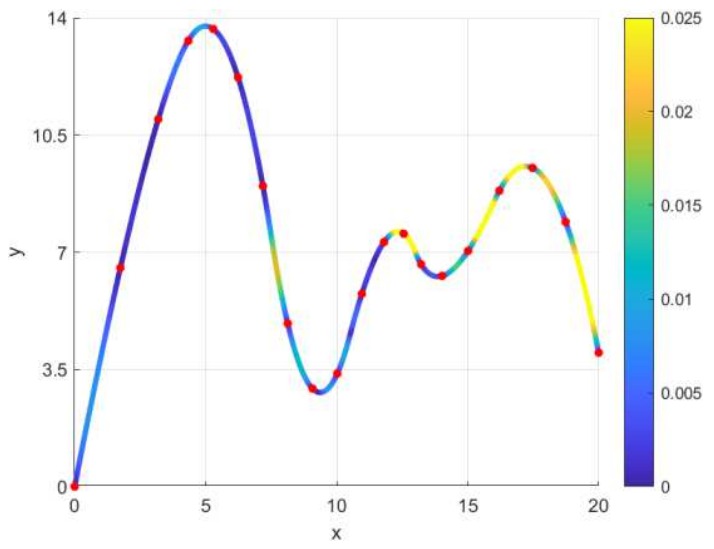

(e) USP

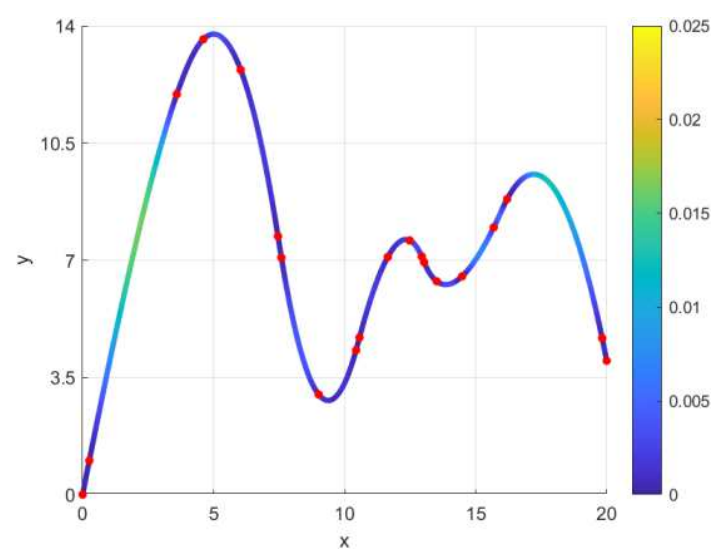

(b) EISD-7

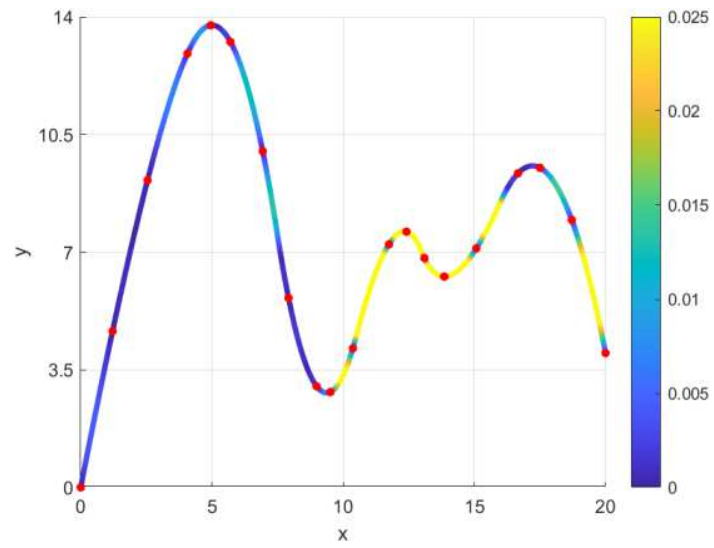

(d) USLC

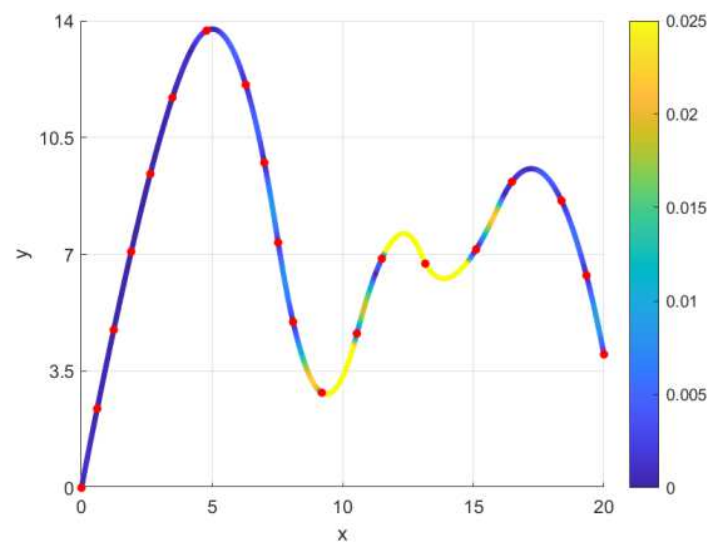

(f) USL

Fig. 13. The reconstruction error and distribution of 20 critical points of different methods

The computing times of the comparison methods are shown in Fig. 14, where it can be seen that the ISD has a long computing time, and the speed of determining the inspection points has increased tremendously from the ISD to the EISD. Therefore, the ISD enhancement was successful. The EISD-L3 $(3,3,3)$ was the least efficient among all methods, so it is suitable for situations where a relatively prolonged process is acceptable. The USP had the shortest computing time among all the methods, because it was the simplest method. The computing times of the EISD, EISD-7, and EISD-L2(3,3) were almost proportional to their reconstruction accuracies. Based on the results presented in Fig. 12-Fig. 14, a suitable method for sampling should be selected as follows:

(1) For smooth curves, the EISD-L2(3, 3) should be selected for time-critical situations; otherwise, 
the EISD-L3(3, 3, 3) should be selected.

(2) For curves with sharp corners, in situations with a few inspection points and where the requirement for computing time is not high, the ISD should be selected. For other situations, the EISD should be selected for the compressed timelines, but if there are many inspection points, the EISD-7 should be selected.

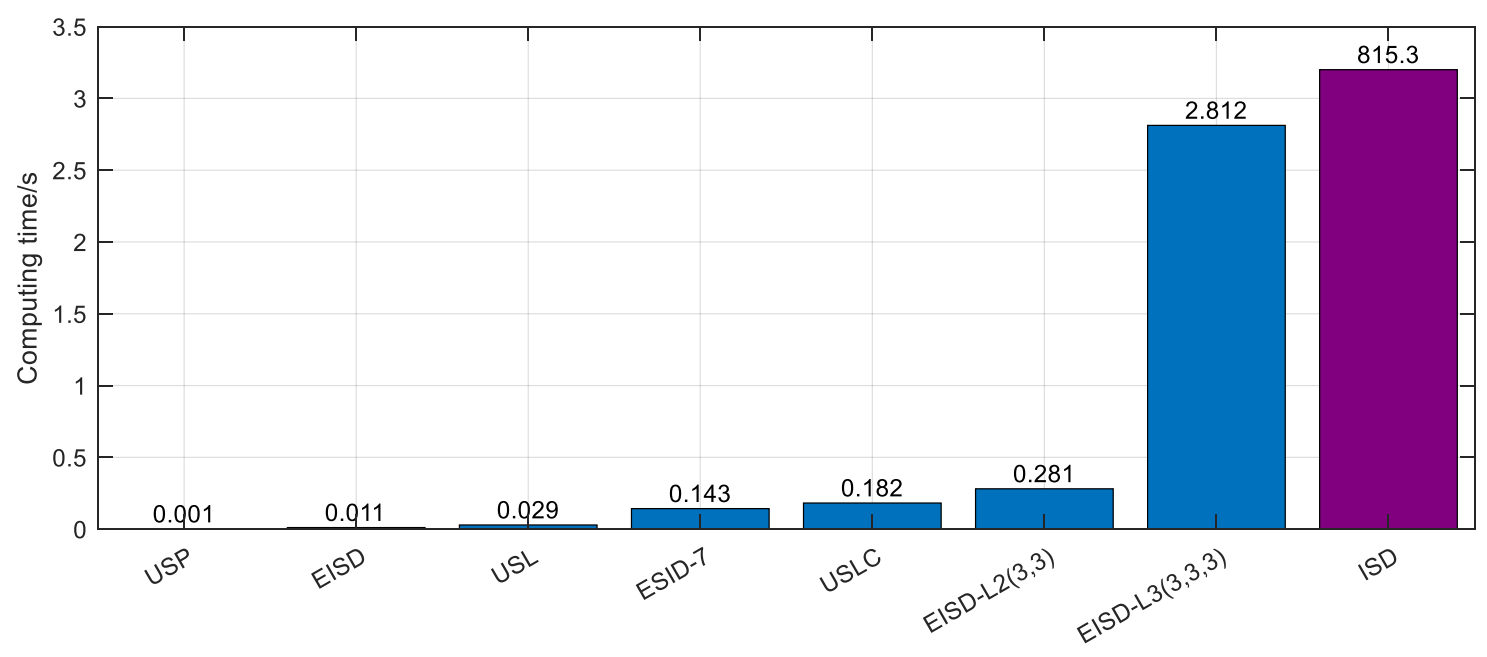

Fig. 14 Computing times of different methods for 20 points

\section{Surface sampling}

This section presents a surface sampling strategy derived from the EISD called the S-EISD, where the inspection points are obtained by the vector product of directions $u$ and $v$. Before presenting this method, a brief introduction to the NURBS surface is given. Also, the S-EISD is compared with several classic sampling methods.

A point on the NURBS surface can be obtained as

$$
\begin{aligned}
\boldsymbol{S}(u, v)= & \frac{\sum_{i=1}^{n} \sum_{j=1}^{m} w_{i, j} B_{i, p}(u) B_{j, q}(v) \boldsymbol{P}_{i, j}}{\sum_{i=1}^{n} \sum_{j=1}^{m} w_{i, j} B_{i, p}(u) B_{j, q}(v)} \\
& u_{1} \leq u \leq u_{n+p+1}, v_{1} \leq v \leq v_{m+p+1}
\end{aligned}
$$

where $p$ is the surface degree in the $u$ direction, $q$ is the surface degree in the $v$ direction, $\boldsymbol{P}_{i, j}$ denote control points, $w_{i, j}$ is the weight of the control point $\boldsymbol{P}_{i, j}$, and $B_{i, p}(u)$ and $B_{j, q}(v)$ are basic functions.

As mentioned previously, the EISD method is based on the reconstruction algorithm of piecewise cubic spline interpolation. Therefore, using bicubic spline interpolation as a surface reconstruct algorithm is necessary in this study. The principle of the bicubic spline interpolation is similar to that of the bilinear interpolation.

Owing to the limitation of bicubic spline interpolation, the surface used in the experiments must meet the requirement that no overlapping area exists after orthogonal projecting the surface onto xoy plane; if the target surface does not satisfy this requirement, it will be divided into several pieces. All the surfaces discussed below satisfy this condition.

Data points used to reconstruct a surface by the bicubic spline interpolation method must satisfy the following requirement: the orthogonal projections of data points onto the xoy plane should distribute in a grid-like shape; particularly, the projections in the same row should have the same $y$ value, and those in the same column should have the same $x$ value, which can be expressed as $x_{1,1}=x_{2,1}=\cdots=x_{k, 1}=x_{1}$, $x_{1,2}=x_{2,2}=\cdots=x_{k, 2}=x_{2}, \ldots$, and $y_{1,1}=y_{1,2}=\cdots=y_{1, k}=y_{1}, y_{2,1}=y_{2,2}=\cdots=y_{2, k}=y_{2}, \ldots$, as 
is shown in Fig. 15.
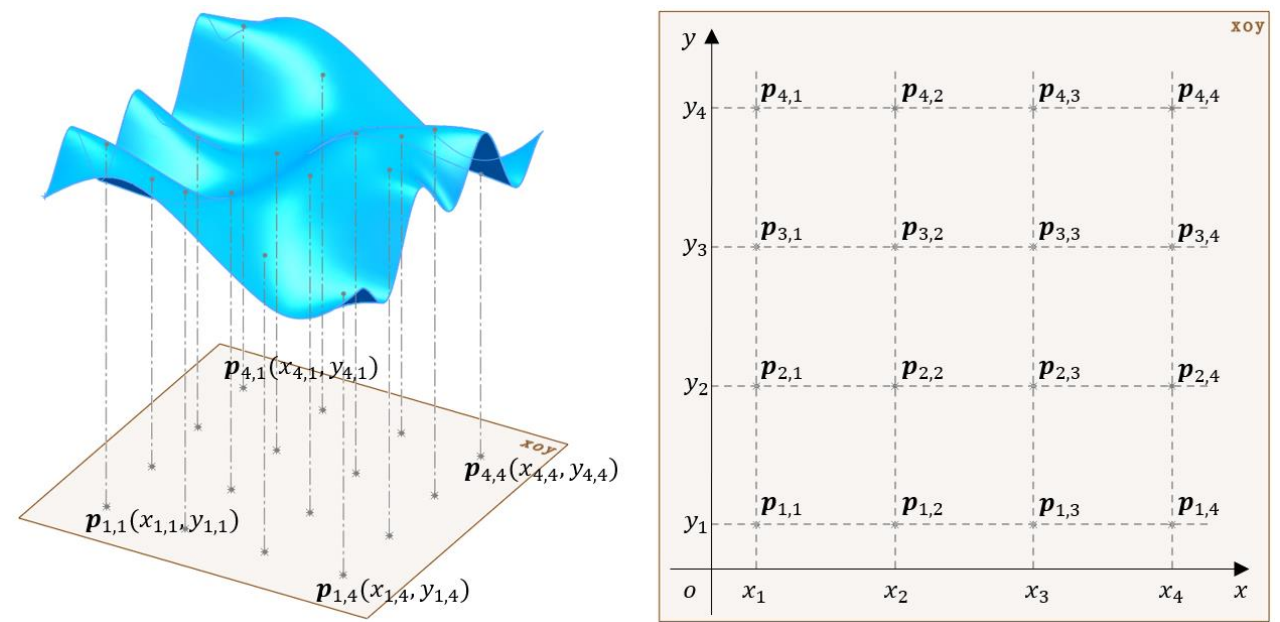

Fig. 15 Layout of data points on the surface

Hence, if the NURBS surface can be expressed as Eq. (18), it will satisfy the above requirement.

$$
\boldsymbol{S}(u, v)=\left\{\begin{array}{c}
x=X(u) \\
y=Y(v) \\
z=Z(u, v)
\end{array} \text { or } \quad \boldsymbol{S}(u, v)=\left\{\begin{array}{c}
x=X(v) \\
y=Y(u) \\
z=Z(u, v)
\end{array}\right.\right.
$$

If the target surface does not satisfy the above requirement, it is transformed as follows.

An arbitrary NURBS surface can be expressed as

$$
\boldsymbol{S}(u, v)=\left\{\begin{array}{l}
x=X(u, v) \\
y=Y(u, v) . \\
z=Z(u, v)
\end{array}\right.
$$

By extracting the nonlinear equations $\left\{\begin{array}{l}x=X(u, v) \\ y=Y(u, v)\end{array}\right.$ from Eq. (19) and solving them, the following result is obtained: $\left\{\begin{array}{l}u=\varphi(x, y) \\ v=\eta(x, y)\end{array}\right.$. If the analytical solution is hard to calculate, the Newton iterative method is used to obtain numerical solutions, and then $\left\{\begin{array}{l}u=\varphi(x, y) \\ v=\eta(x, y)\end{array}\right.$ is substituted into $z=Z(u, v)$, so $z=Z(\varphi(x, y), \eta(x, y)) \quad$ is obtained, and Eq. (19) is transformed into $\boldsymbol{S}(u, v)=$ $\left\{\begin{array}{l}x=u \\ y=v\end{array}\right.$

$$
z=Z(\varphi(u, v), \eta(u, v))
$$

, which satisfy the requirement of Eq. (18), where $x$ represents the parameter $u$, and $y$ represents the parameter $v$.

Another relatively easy way to transfer an arbitrary surface to a compliant surface is picking many points from the surface by the USP and reconstructing a surface by the algorithm implemented in the fit function and "cubicinterp" fit mode of MATLAB software, thus obtaining a surface with the format of $z=z(x, y)$. If the accuracy requirement is high, more points should be selected to reconstruct the surface using MATLAB software, and this process consumes less than $1 \mathrm{~s}$.

The reason why the fit function with the fit mode of "cubicinterp" in MATLAB software is not used as a default reconstruction algorithm is that it is not the standard bicubic spline interpolation. 


\subsection{Extended approach}

For a free-form surface $\boldsymbol{S}(u, v)$ that can be expressed by Eq. (18), $\boldsymbol{S}\left(u_{\ell}, v\right)$ or $\boldsymbol{S}\left(u, v_{\ell}\right)$ represents a planar curve, in which $u_{\ell}$ and $v_{\ell}$ are constant values. The previously proposed curve sampling methods can be applied to $\boldsymbol{S}\left(u_{\ell}, v\right)$ or $\boldsymbol{S}\left(u, v_{\ell}\right)$.

The goal of surface sampling is to obtain two vectors in the $u$ and $v$ directions, $u_{\text {aim }}=\left[u t_{1}, u t_{2}, \cdots u t_{N_{u}}\right]$, and $v_{\text {aim }}=\left[v t_{1}, v t_{2}, \cdots v t_{N_{v}}\right]$, respectively. All combinations of the values in $u_{\text {aim }}$ and $v_{\text {aim }}$ denote the parameter coordinates of inspection points, that is Points $=\left\{\boldsymbol{S}\left(u_{i}, v_{j}\right) \mid u_{i} \in\right.$ $\left.u_{\text {aim }}, v_{j} \in v_{\text {aim }}\right\}$.

To take the global characteristics of the surface into consideration, many curves are extracted from the surface, and their sampling points are obtained by the EISD; then, the average parameter values of the corresponding points are denoted as targets.

This work can be divided into two directions: $u$ and $v$. Assume that the surface can be expressed as $\boldsymbol{S}(u, v)=\left\{\begin{array}{c}x=X(u) \\ y=Y(v) \\ z=Z(u, v)\end{array}\right.$ or has been transformed into this form; then, in the $u$ direction, select several $v$ parameters to obtain a series of curves $\boldsymbol{S}\left(u, v_{1}\right), \boldsymbol{S}\left(u, v_{2}\right), \cdots$, as shown in Fig. 16.

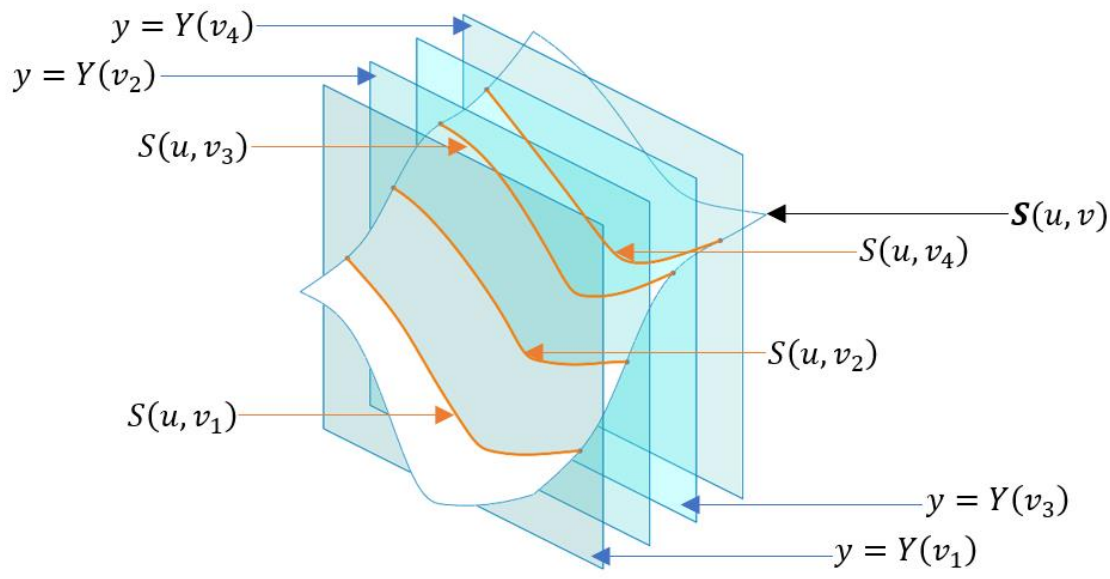

Fig. 16 The intersection lines

Next, obtain the sampling points on the intersection lines by the EISD, extract $u$ parameters of the first point on each intersection line, and calculate the average value as $u t_{1}$, as shown in Fig. 17. Similarly, obtain $u t_{2}, u t_{3}, \cdots$. Then, the target vector $u_{\text {aim }}$ is obtained. The other target vector $v_{\text {aim }}$ can be obtained in a similar way.

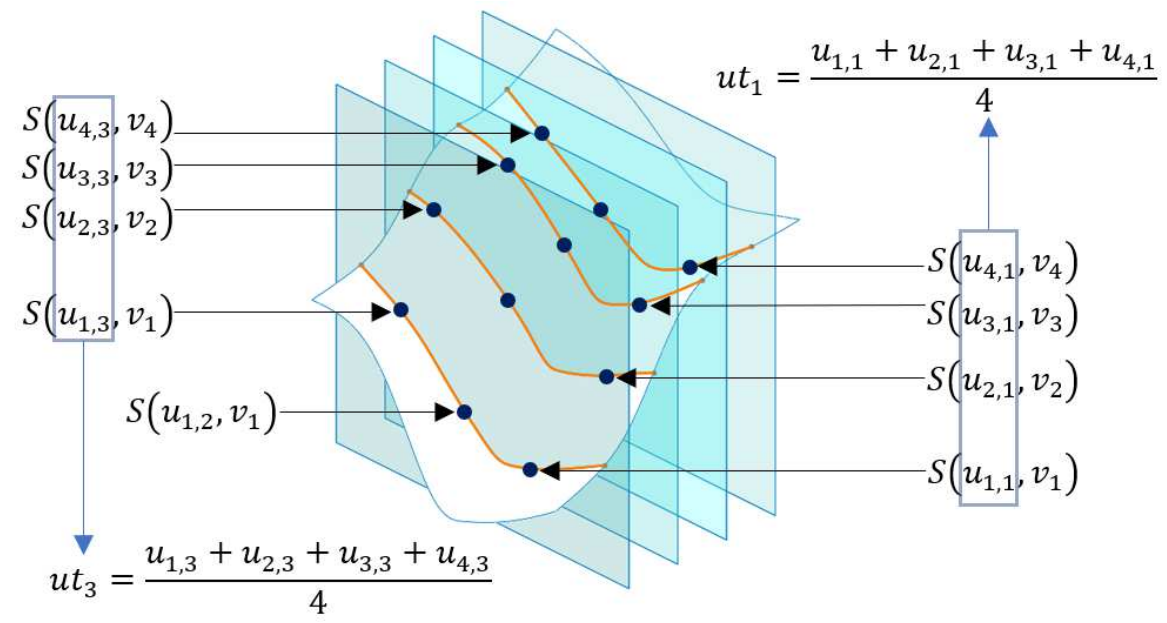


Fig. 17 Computation method of the target parameter

\subsection{S-EISD algorithm}

Assume the parametric space of the NURBS surface is expressed as $u \in\left[u_{1}, u_{n+p+1}\right], v \in$ $\left[v_{1}, v_{m+p+1}\right]$, and the numbers of inspection points in the $u$ and $v$ directions are $N_{u}$ and $N_{v}$, respectively. Then, the sampling process of the S-EISD is as shown in Algorithm 3.

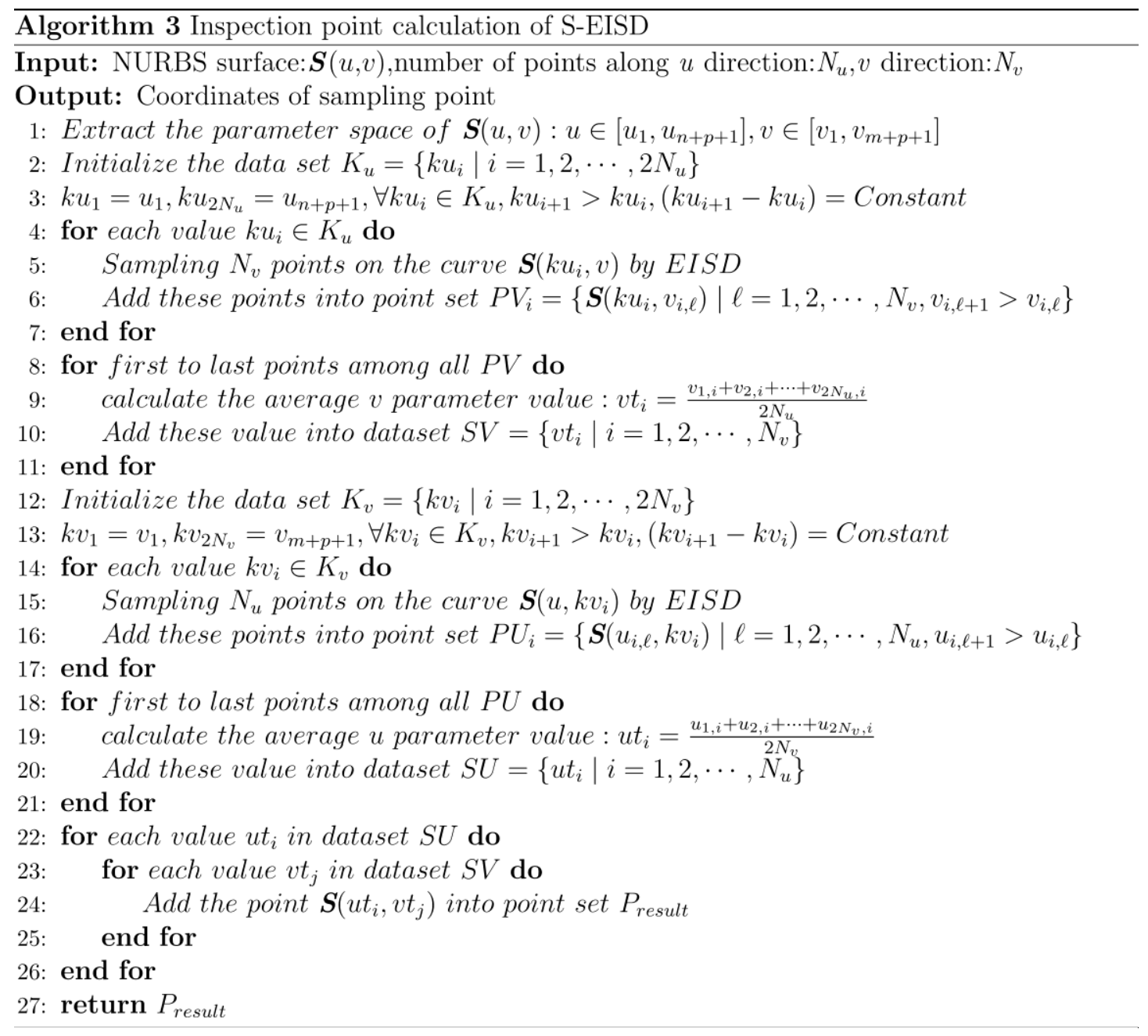

The main idea of the S-EISD is to simplify surface sampling to curve sampling. To obtain more information on a surface, many lines are used to sample points from the surface, and then the corresponding point parameters are averaged as the target parameter. Generally speaking, the more lines are used, the higher accuracy will be obtained, but the computing time will increase. In this study, a relatively medium number of lines are used, which can guarantee a good result, but will not significantly increase the computing time.

\subsection{Surface sampling experiments}

In this section, four sampling methods are compared on three NURBS surfaces with different complexities. The ISD method has not been extended to surface sampling because this method consumes too much time, so it is difficult to be applied. The reconstruction algorithm is the bicubic spline interpolation; the number of inspection points is $N_{u} \cdot N_{v}$, where $N_{u}$ denotes the number of inspection points in the $u$ direction, and $N_{v}$ denotes the number of inspection points in $v$ direction.

The comparison surface sampling methods are as follows: S-EISD, USP, uniform sampling of 
accumulative marginal areas (USE) [2], and uniform sampling of accumulative marginal areas and mean curvature (USEC) [2].

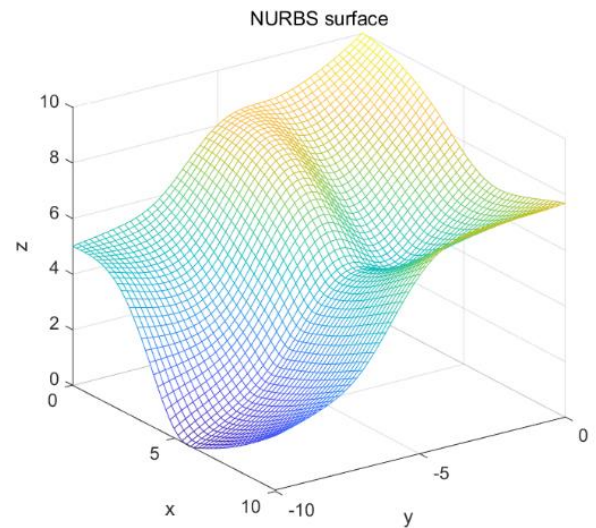

(a) NURBS surface

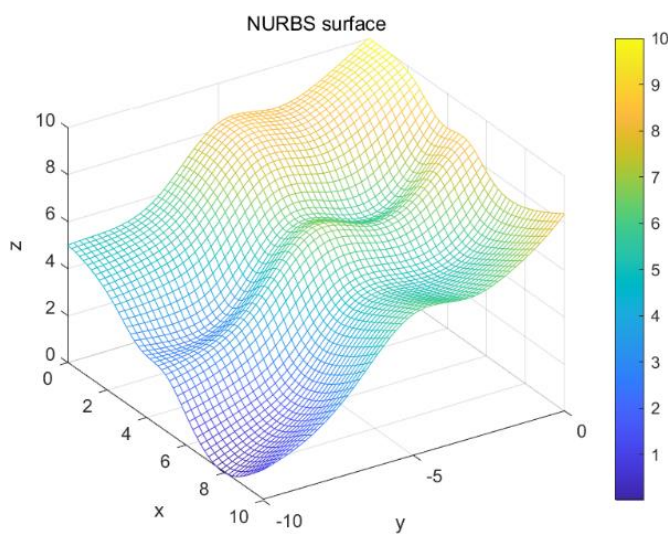

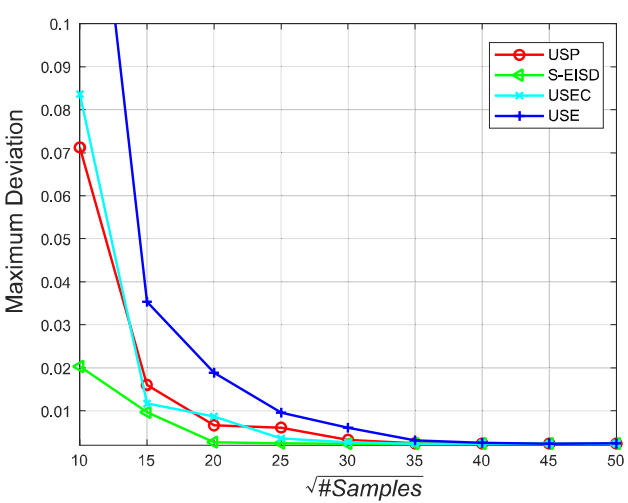

(b) Maximum deviation as a function of $\sqrt{\# \text { samples }}$

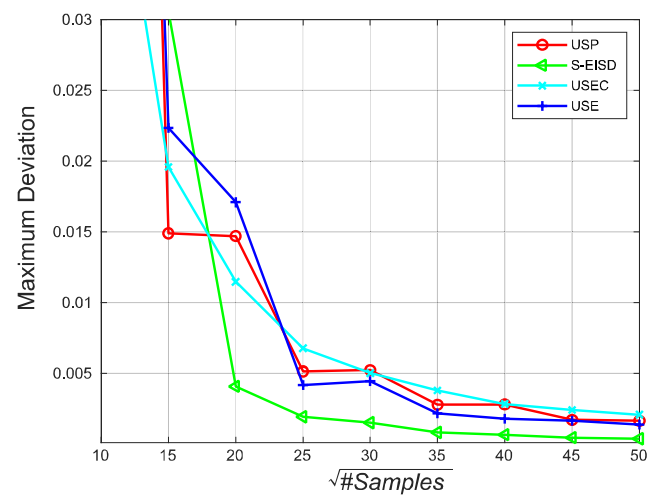

(d) Maximum deviation as a function of $\sqrt{\# \text { samples }}$

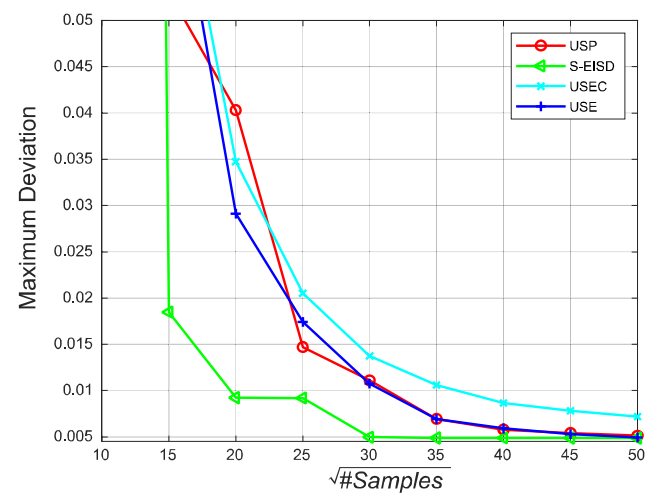

(f) Maximum deviation as a function of $\sqrt{\# \text { samples }}$

Fig. 18 The NURBS surfaces used in the comparison and the obtained reconstruction errors of different methods

The maximum deviations of the four sampling methods are shown in Fig. 18(b), 18(d), and 18(f), where it can be seen that the proposed method S-EISD performed best among all the methods. Therefore, it can be concluded that the S-EISD is effective for various complex surfaces. By extracting a series of curves on the surface, the global features of the surface can be successfully obtained by S-EISD. It should be noted that the performance of the USEC was not satisfactory, which differs from the conclusion drawn by Pagani and Scott [2], which could be due to different reconstruction algorithms and evaluation metrics. 
The reconstruction error and inspection point distribution of the sampling methods are presented in Fig. 19, where it can be seen that the reconstruction results of the USP and USE were unsatisfactory at complex areas because these methods considered only the parameter and area of the surface, respectively. The proposed strategy S-EISD achieved the best result among all the methods. Under the same number of inspection points, the reconstruction error was greatly reduced by the S-EISD compared to the other methods. The USEC performed better than the USP and USE, but there was a problem of large error in the complex areas, so placing more inspection points at areas with a high curvature could be better.

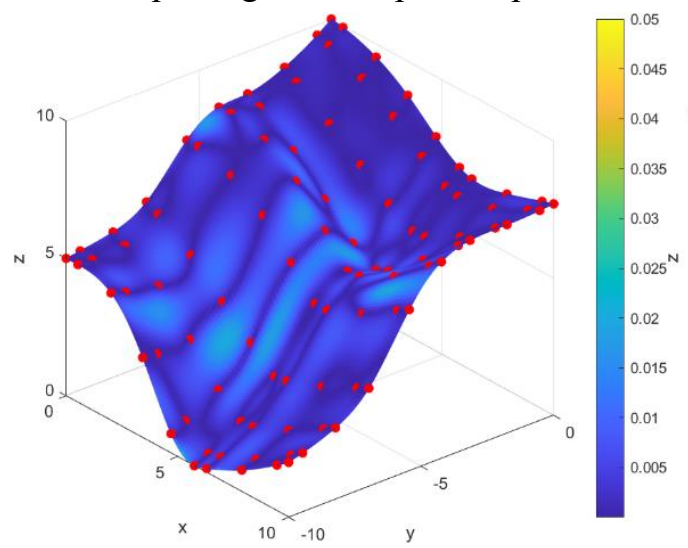

(a) S-EISD (the proposed method)

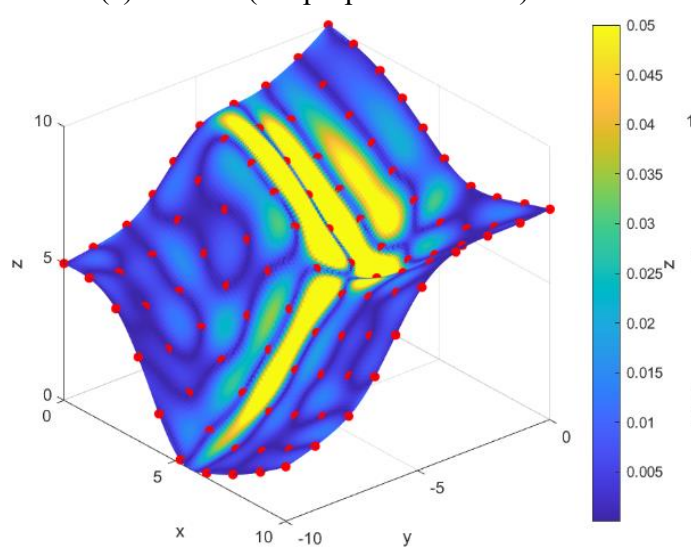

(a) USE

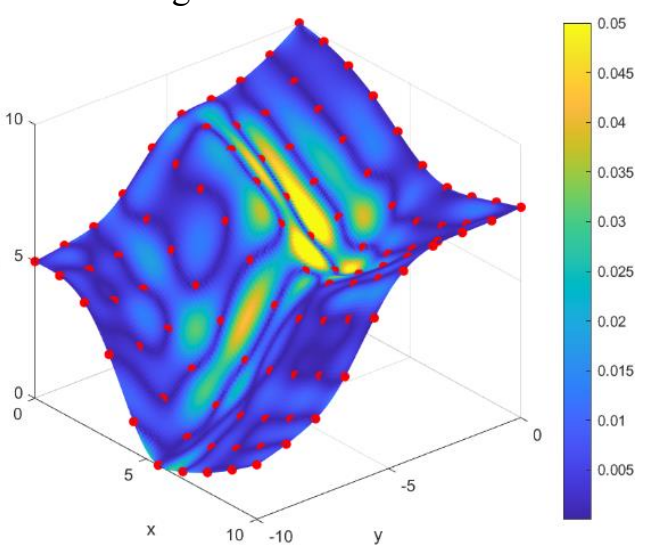

(b) USP

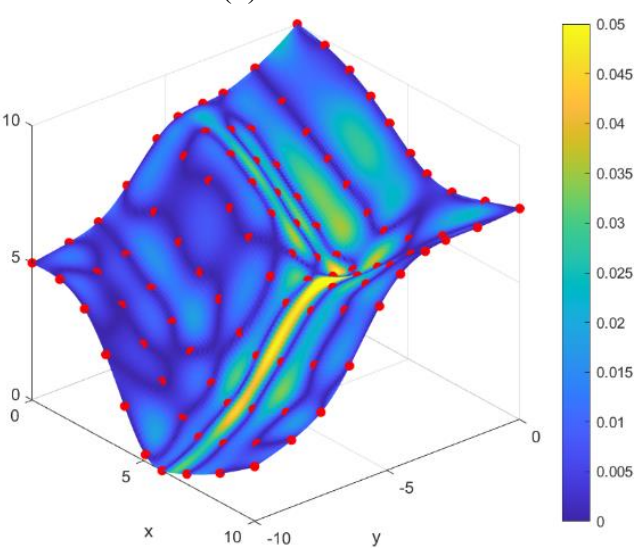

(b) USEC

Fig. 19 Inspection points and reconstruction errors of different methods

\section{Conclusion}

This article proposes two methods for curve sampling and several derivative methods. The first sampling method, the ISD, achieves high reconstruction accuracy but consumes too much time. This method is based on the real-time reconstruction error and removes the less significant points from the initial point set iteratively. The second sampling method, the EISD, is inspired by the ISD. In this method, an approximate algorithm is used to perform point deletion from the initial point set, thus decreasing the computing time significantly while achieving the reconstruction accuracy similar to that of the ISD. Next, the relationship between the quality and quantity of initial points and the reconstruction accuracy of the EISD is studied, and based on the above research, several derivative methods are proposed; the reconstruction accuracies of these derivative methods exceed that of the ISD. The proposed methods are compared with several classic sampling methods, and the experimental results prove that the proposed 
methods perform well. For surface sampling, a method extended from the previous curve sampling strategy is proposed, and its reconstruction error is compared with those of several classic surface sampling methods. The experimental results show that the proposed method performs best among all the methods; therefore, the quality of inspection points has been improved.

In this study, the initial point set is obtained by either the USP or the proposed method, but selecting initial points using other strategies may provide a different result. In this study, the ratio of auxiliary points is fixed at 0.1 , but for different curves, 0.1 may not be the best choice; however, a relatively good result can be obtained. For the reconstruction algorithm, the piecewise cubic spline interpolation is selected as a default, but other reconstruction algorithms could be analyzed in a similar way. We will also analyze other reconstruction algorithms to obtain better sampling methods in the future.

\section{Declarations}

\section{Funding}

This paper is supported by the National Natural Science Foundation of China (Grant No. 61972220), National Defense Basic Scientific Research Program (JCKY2018208B014).

\section{Conflicts of interest/Competing interests}

Not applicable

\section{Availability of data and material}

Not applicable

\section{Code availability}

Not applicable

\section{Authors' contributions}

Xi Cheng, Long Zeng designed all technique routes and experiments included in this study, wrote and modified this manuscript. Haoyu Jiang and Xueping Liu Conducted the experiments and data analysis work.

\section{Ethics approval}

The authors declare that this manuscript was not submitted to more than one journal for simultaneous consideration. Also, the submitted work is original and not have been published elsewhere in any form or language.

\section{Consent to participate}

The authors declare that they participated in this paper willingly.

\section{Consent for publication}

The authors declare to consent to the publication of this paper. 


\section{Reference}

1. Li S, Zeng L, Feng P, Li Y, Xu C, Ma Y (2019) Accurate compensation method for probe pre-travel errors in on-machine inspections. International Journal of Advanced Manufacturing Technology 103:2401-2410

2. Pagani L, Scott PJ (2018) Curvature based sampling of curves and surfaces. Computer Aided Geometric Design 59:32-48

3. Elkott DF, Elmaraghy HA, Elmaraghy WH (2002) Automatic sampling for CMM inspection planning of free-form surfaces. International Journal of Production Research 40:2653-2676

4. Hernandez-Mederos V, Estrada-Sarlabous J (2003) Sampling points on regular parametric curves with control of their distribution. Computer Aided Geometric Design 20:363-382

5. Wang J, Jiang X, Blunt LA, Leach RK, Scott PJ (2012) Intelligent sampling for the measurement of structured surfaces. Measurement Science and Technology 23

6. Lee G, Mou J, Shen Y (1997) Sampling strategy design for dimensional measurement of geometric features using coordinate measuring machine. International Journal of Machine Tools \& Manufacture 37:917-934

7. Abdulhameed O, Mian SH, Al-Ahmari A, Alkhalefah H (2020) Patch and curvature specific estimation of efficient sampling scheme for complex surface inspection. International Journal of Advanced Manufacturing Technology 110:3407-3422

8. He G, Jia H, Guo L, Liu P (2012) Adaptive Sampling Strategy for Free-form Surface Based on CAD Model. In: Tan RH, Sun J, Liu QS (eds) Automatic Manufacturing Systems Ii, Pts 1 and 2, pp 541-544

9. Lu L, Zhao S (2019) High-quality point sampling for B-spline fitting of parametric curves with feature recognition. Journal of Computational and Applied Mathematics 345:286-294

10. Obeidat SM, Raman S (2009) An intelligent sampling method for inspecting free-form surfaces. International Journal of Advanced Manufacturing Technology 40:1125-1136

11. Chen Y, Peng C (2017) Intelligent adaptive sampling guided by Gaussian process inference. Measurement Science and Technology 28

12. He G, Sang Y, Pang K, Sun G (2018) An improved adaptive sampling strategy for freeform surface inspection on CMM. International Journal of Advanced Manufacturing Technology 96:1521-1535

13. Lu W, Pagani L, Zhou L, Liu X, Wang J, Leach R, Jiang X (2019) Uncertainty-guided intelligent sampling strategy for high-efficiency surface measurement via free-knot B-spline regression modelling. Precision Engineering-Journal of the International Societies for Precision Engineering and Nanotechnology 56:38-52

14. Mansour G (2014) A developed algorithm for simulation of blades to reduce the measurement points and time on coordinate measuring machine (CMM). Measurement 54:51-57

15. Yu M, Zhang Y, Li Y, Zhang D (2013) Adaptive sampling method for inspection planning on CMM for free-form surfaces. International Journal of Advanced Manufacturing Technology 67:1967-1975

16. Feng G, Ziyue P, Xutao Z, Yan L, Jihao D (2021) An adaptive sampling method for accurate measurement of aeroengine blades. Measurement: Journal of the International Measurement Confederation 173

17. Han Z, Liu S, Yu F, Zhang X, Zhang G (2017) A 3D measuring path planning strategy for intelligent CMMs based on an improved ant colony algorithm. International Journal of Advanced Manufacturing 
Technology 93:1487-1497

18. Zahmati J, Amirabadi H, Mehrad V (2018) A hybrid measurement sampling method for accurate inspection of geometric errors on freeform surfaces. Measurement 122:155-167 